\title{
MedienPädagogik
}

Zeitschrift für Theorie und Praxis der Medienbildung

Jahrbuch Medienpädagogik 18: Ästhetik - Digitalität - Macht

Herausgegeben von Benjamin Jörissen, Claudia Roßkopf, Klaus Rummler,

Patrick Bettinger, Mandy Schiefner-Rohs, Karsten D. Wolf

\section{time (space) matters}

\section{Mediatisierte Zeit(räume) in Tutorial-Videos}

\author{
Nadia Bader ${ }^{1} \bullet$ und Notburga $\operatorname{Karl}^{2} \bullet$ \\ ${ }^{1}$ Pädagogische Hochschule Freiburg \\ ${ }^{2}$ Universität Bamberg
}

\section{Zusammenfassung}

Am Beispiel von Tutorial-Videos als digitale Lehr- und Lern-Medien fragt der Beitrag nach deren medialen Eigenheiten und medial durchwirkten Bildungspotenzialen. Inwiefern schwingen mediale Übersetzungsprozesse, medial vermittelte Momente des Zeigens und Erklärens implizit in der Ausgestaltung solcher Videos mit? Inwiefern werden sie explizit genutzt? Und welche Bildungspotenziale birgt ein medial sensibilisierter Umgang mit (audio-)visuellen Gestaltungsmöglichkeiten? Im Rahmen aktueller Lehrentwicklungen (Blended und Distance Learning) lohnt sich eine genauere Untersuchung insbesondere bildungsrelevanter, medienästhetischer Dimensionen bestimmter (Lehr-/Lern-)Medien und Tools. Mit Erfolgsgarantie werbend - z. B. zum perspektivisch 〈richtigen〉 Zeichnen suggerieren Tutorial-Videos Verständlichkeit, Machbarkeit und einen schnellen, selbstbestimmten Zugriff auf deren Lerninhalte (z. B. durch Fast Forward, Pause, Wiederholung). Zugleich kann die damit einhergehende Effektivitätslogik einer unreflektierten Reproduktion impliziter normativer Vorstellungen Vorschub leisten. Die Frage nach dem Wie der Darstellung kann dahingehend eine medial sensibilisierte, repräsentationskritische Sichtweise eröffnen. Beispielsweise kann sich 〈laienhafte〉 Nachlässigkeit in einer (irritierenden) Bildstörung als bildend zeigen, wenn sie die Aufmerksamkeit von Rezipierenden auf deren eigene Wahr- 
nehmung lenkt und sie dazu auffordert, sich zu erklärten und gezeigten Inhalten (bewusster) ins Verhältnis zu setzen, satt vorschnell einer (Verstehensillusion〉 $z u$ erliegen. Im Sinne einer transformatorischen Bildungstheorie sind solche Wahrnehmungsbrüche potenziell eher ermächtigend als beispielsweise die symbolische Form der Perspektive selbst und ermöglichen eine kritische Auseinandersetzung damit. Im vorliegenden Text-Bild-Beitrag nutzen wir distanzierendkritische sowie künstlerisch-experimentelle Methoden, um medial bedingte Bildungspotenziale in Tutorial-Videos aufzudecken und weiterzudenken.

\title{
time (space) matters. Mediated Time (Spaces) in Video Tutorials
}

\begin{abstract}
This contribution focuses on video tutorials used for teaching and learning. It looks at their medial properties and probes their (media-specific) educational potential. We enquire both into some of the implicit features of such tutorials e.g., translations and transformations between media, or moments of showing and explaining with their distinct medial properties - as well as their explicit use: specifically, the educational potential they may yield if audio-visual means of designing and creating are employed in media-sensitive fashion. Given the current developments in teaching practices (such as blended and distance learning), a more detailed investigation of the aesthetic dimension of these teaching tools seems to be indicated. Video tutorials often guarantee their users' successful acquiring of skills, e.g. of drawing a perspective (correctly); and they implicitly promise an easy grasp of the subject, practical feasibility and quick, self-determined access to learning content (e.g. by fast-forwarding, pausing or replaying content). At the same time, they promote a logic of effectiveness which may hide normative ideas that users uncritically adopt and reproduce. Looking into their means of presentation may allow for a media-sensitive critique of their representational logic. 〈Amateur〉 negligence may, for example, turn out to be educational - if unsettled/-ling images protect the users of such tutorials from the illusion of a mere passive 〈understanding - and instead direct the attention to their own perception and their own position in relation to the subjects that are
\end{abstract}


being presented and explained to them. As part of a transformative educational theory, such perceptual disturbances are potentially more empowering than, for example, knowledge about perspective as a symbolic form as such. They invite a critical engagement with the latter. In our contribution, we will use both conventional critical methods and more experimental artistic strategies to explore the educational potential of video tutorials.

\section{Eine experimentelle Gegenüberstellung}
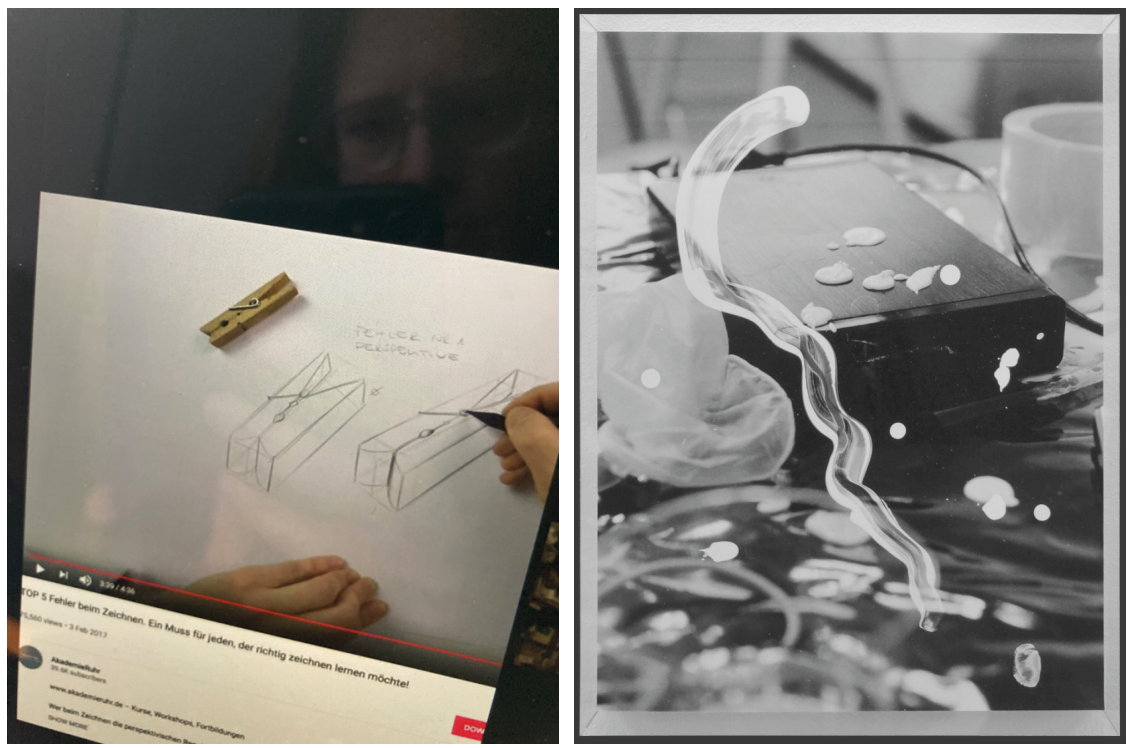

Abb. 1: Bildschirmfoto mit Spiegelung (Foto: Notburga Karl).

Abb. 2: Vaka Olsen 2014, «Sunscreenpill», ๑ Kunstverein Nürnberg.

Der vorliegende Beitrag möchte in der Zeit forcierter Digitalisierung den Blick für Zusammenhänge schärfen, die nicht nur aus kunstpädagogischer Sicht wichtig sind: die Medienästhetik. Dazu knüpft er an das Labor «time (space) matters - Mediatisierte Zeit in Tutorial-Videos» an, das anlässlich der digitalen Tagung «Ästhetik - Digitalität - Macht» stattfand. Inspiriert von der experimentell, performativ und interaktiv angelegten und (audio-)visuell ausgestalteten Laborarbeit, gemeinsam mit den Teilnehmenden, spitzten wir für diesen Beitrag jene Fragen zu, die wir aus 
medientheoretischer sowie produktionsästhetischer Sicht für das thematische Spektrum des Tagungsdiskurses interessant finden. Andererseits fokussieren wir jene Fragen, die sich gut im Medium des hier gegebenen Beitragsformats (digital, schriftlich, bildlich) diskutieren lassen.

Im Folgenden beschäftigen wir uns exemplarisch mit Tutorial-Videos als (Lehr-, Lern- und Bildungs-)Medium, das sich zunehmender Beliebtheit erfreut und inzwischen in unübersichtlicher Vielzahl online zugänglich ist (vgl. Bader 2022/i. V.). Wir gehen davon aus, dass (digitales bzw. digital gestütztes) Lernen - beispielsweise mit Tutorial-Videos - von damit verbundenen medialen Eigenheiten massgeblich beeinflusst und geradezu durchwirkt wird. So erscheint es lohnend, diese Eigenheiten mit Blick auf konkrete Phänomene und Wirkweisen sowie damit verbundene Möglichkeiten und Grenzen genauer zu betrachten und in der aktuellen Lehrentwicklung (z. B. Distance/Blended Learning) zu berücksichtigen. Über die funktionale und didaktische Ebene eines Mediums oder Tools hinaus gilt es, dabei insbesondere dessen medienästhetische Prämissen zu beachten.

Konkret untersuchen wir die mediale Ausgestaltung von (ausgewählten) Tutorial-Videos, um medial bedingte bzw. beeinflusste Bildungspotenziale herauszuarbeiten, denn die je spezifische Nutzung (audio-)visueller Möglichkeiten bei der Gestaltung von Videos kann den zu vermittelnden Inhalten zugutekommen oder ihnen entgegenstehen. Mediale Potenziale können explizit genutzt werden oder implizit und unbeachtet bleiben. Im Rückgriff auf Christoph Koller und Ursula Brandstätter (vgl. Koller 2018; Brandstätter 2013) nehmen wir an, dass mediale Übersetzungsprozesse als solche bildende Potenziale aufweisen. Diesen gilt daher unsere besondere Aufmerksamkeit.

Ein Anliegen unseres Beitrags ist zudem, die Wahrnehmung des eigenen (medial geprägten) Wahrnehmens als solche zu thematisieren. Neben einem kritisch-analytischen Vorgehen erscheinen uns dafür auch ästhetisch-explorative, spielerische Zugänge gewinnbringend, wie wir sie u. a. im Tagungslabor erprobt haben. Diese können dazu beitragen, dass wir unseren Untersuchungsgegenstand in einem nochmals anderen Licht und sensibilisiert für mediale Zusammenhänge betrachten können (vgl. Bader und Götsch 2020; Karl 2022). 
Wir schliessen damit an zeitgenössische Bestandsaufnahmen an (v. a. Jörissen und Meyer 2015): In unserer medial veränderten Welt und deren Infrastruktur haben sich auch Subjektwerdungsprozesse in Interaktion mit Medien längst verändert. So braucht es zu deren Bewusstwerdung eine eigene Wahrnehmungssensibilisierung, die zugleich eine rein handlungstheoretische Perspektive um eine «relationale, sozio-kulturelle» Perspektive erweitert (Zahn 2020, 213). Dieses Ansinnen soll im Folgenden (methodisch) mitgedacht werden.

Der Beitrag geht zunächst auf die thematische Auswahl der zu untersuchenden Tutorial-Videos ein, die sich kunstpädagogisch begründen lässt und mit unserem Erkenntnisinteresse an medialen Eigenheiten zusammenhängt. Im Anschluss wird das methodische Vorgehen, das distanzierend-kritisch sowie ästhetisch-explorativ ausgerichtet ist, theoretisch grundiert. Dann folgt die Untersuchung konkreter Beispiele, an denen wir nach und nach aufzeigen und erörtern, wie eine für Medialität sensibilisierte Zugangsweise aussehen und welche Erkenntnisse sie zeitigen kann. So werden wir u. a. exemplarisch zeigen, welche (medial bedingten) Bildungspotenziale in den untersuchten Tutorial-Videos angelegt sind, inwiefern diese implizit bleiben und erst durch die Analyse aufgedeckt werden, oder aber bereits im Videoformat selbst genutzt werden.

\section{Erfolg und Fragwürdigkeit perspektivischer Darstellungskonventionen}

In unserer Untersuchung ziehen wir als Beispiele Tutorial-Videos heran, die sich mit dem Gegenstand perspektivischen Zeichnens beschäftigen. Das Thema Perspektive fokussiert die Auseinandersetzung mit spezifischen, regelgeleiteten Formen der zeichnerischen Raumdarstellung auf der zweidimensionalen Fläche und kann im kunstpädagogischen Diskurs nach wie vor als aktuell sowie fachhistorisch 〈aufgeladen〉 gelten. Eine kritische Betrachtung bietet sich an (vgl. Hoffmann 2015, 357ff.), da es bei dem Thema Perspektive bildungstheoretisch um einen komplexeren Zusammenhang von Subjekt und Welt geht, als es diese Darstellungsregeln je vermitteln könnten. Gerade das Thema der Perspektive bräuchte eigentlich eine mehrperspektivische Kontextualisierung. Im Streben nach Eindeutigkeit 
wird oft der Unterschied zwischen einer mathematisch konstruierbaren Sehkonvention und einer zur Darstellung drängenden visuellen Wahrnehmungserfahrung von Raum ignoriert.

Im Netz finden sich zudem viele Tutorial-Videos, die sich mit dem Thema Perspektive im ersteren Sinne beschäftigen. Die Popularität solcher Videos verweist auf eine gewisse Dringlichkeit der Thematik und auf eine Hoffnung, ein Begehren seitens der Rezipierenden, dass Tutorial-Videos ermächtigend wirken und helfen, Probleme im Zusammenhang mit Raumdarstellung zu lösen. Auffällig ist, wie viele Videos den Fokus auf linearperspektische Konstruktionen mit ein, zwei oder drei Fluchtpunkten legen. Zugleich werden unter dem Begriff Perspektive aus fachlicher Sicht verschiedene Darstellungsformen zusammengefasst, wobei die Arbeit mit ein, zwei oder drei Fluchtpunkten jeweils einen Sonderfall darstellt (vgl. Bader 2022/i. V.; Asisi 2020). Weiter gefasst ist die perspektivische Darstellung eine von zahlreichen Möglichkeiten, sich bildnerisch mit Raumdarstellung zu beschäftigen. Für den kunstdidaktischen Diskurs lässt sich im Anschluss an Stephanie Marr (2015) und Katja Hoffmann (2015) aufzeigen, wie eine Fokussierung auf fluchtpunktperspektivische Raumdarstellungen zwar (fach-)historisch begründbar ist, zugleich aber eine problematische Engführung darstellen kann, insbesondere wenn es in einem zeitgenössischen (Kunst-)Unterricht um die Vermittlung und Förderung grundlegender, überfachlich relevanter bildsprachlicher sowie medialer Kompetenzen geht.

Es handelt sich beim fluchtpunktperspektivischen Zeichnen also um einen aktuell populären Sonderfall, dessen Popularität und Wirkmacht sich (fach-)historisch sowie kulturell nachweisen lässt, die jedoch zugleich kritisch zu befragen ist. Der Anspruch an eine 〈gute〉 Zeichnung wird allzu oft mit dem Anspruch an eine (perspektivisch richtige) Zeichnung gleichgesetzt. Gerne wird auch die Historizität und die Bedingtheit dieser Darstellungskonvention übersehen, was bereits eine Wahrnehmungsverschiebung und eine höchst spezifische darstellungsbezogene Engführung mit sich bringt. Bereits das Zeichnen ist ein medialer Übersetzungsprozess einer raumzeitlichen Situation auf die zweidimensionale Fläche des Papiers, der sich bei der (zentral-)perspektivischen Konstruktion als symbolische Form fortschreibt. Das aktivierte räumliche Vorstellungsvermögen bei 
dieser Darstellungskonvention ist lernbar und sehr überzeugend, ein optischer Trick, was deren Popularität begründet. Der Blick aus dem Fenster, der die Zentralperspektive innerhalb eines rechtwinkligen Formats fundiert und in einer strengen Rasterlogik organisiert (vgl. Pazzini 1992; Blum und Hartle 2010), geht mit der technischen Entwicklung der Optik einher, sodass dieser Fensterblick dem (begehrlichen) Blick der Kamera sehr nahekommt (vgl. Hitchcock 1954, «Das Fenster zum Hof»). Dass dabei Brennweiten, Zooming, Einäugigkeit, Augenbewegung, die Performanz des Sehvorgangs oder gar andere Raumauffassungen und Darstellungslogiken (vgl. Hoffmann 2015) ausser Acht gelassen werden, schmälert den Erfolg dieser Darstellungskonvention nicht, gelingt es ihr doch, ohne Anschauung und Vergewisserung anhand eines Gegenübers immer neue virtuelle Raumkonstrukte glaubhaft zu entwerfen. Selbst wenn diese Raumkonstrukte verzerrt erscheinen und so einen potenziell irritierenden Kontrast zur visuellen Wahrnehmung in physischen Räumen bilden, vermittelt sich eine Raumauffassung im Sinne der Raster- und Behälterlogik immer aufs Neue (vgl. die 3D-Grafik in Computerspielen; vgl. Steyerl 2015, «Factory of the Sun»). Panofsky bezeichnet die (Zentral-)Perspektive als symbolische Form (Panofsky 1927). Doch nicht nur die Historizität ihres Erfolgs macht diese Darstellungsform machtvoll und begehrenswert, sondern auch die ideal konstruierten Standpunkte, wie sie die zumeist linear angelegte Zentralperspektive für die schauenden Subjekte vor dem Bild ermächtigend vorsieht (vgl. Crary 1992). Diese werden mit Mitteln der Lineatur als Blicklenkung (z. B. über Formgrenzen von Dachgesimsen, Fensterkanten, Teppichen, Bodenfliesen etc.) vor dem Bild «zurechtgerückt». Durch das Ineinsfallen von Augenhöhe und Fluchtpunkt ist ein ideal gesetzter Betrachtendenstandort durch den Fluchtpunkt im Bild vorgegeben. Schauende Subjekte können dann in den virtuellen Bildraum vordringen, ihn visuell und mental erobern. Künstlerinnen und Künstler gehen deswegen mit der Zentralperspektive und fluchtenden Bildelementen immer wieder in einem medienkritischen Sinne manipulativ um (vgl. Laleg 2021).

Vor diesem Hintergrund erscheint es interessant und brisant, uns gerade am Thema Perspektive ästhetisch reflektierend sowie nahe an den Phänomenen medialer Erscheinungen und Darstellungen abzuarbeiten, um so die eigene Wahrnehmung zu sensibilisieren und die selbst 
gemachten Erfahrungen angemessen zu kontextualisieren. Mit einem medienkritischen Zweifel an der Darstellungskonvention lässt sich ferner die Frage im übertragenen Sinne auf das Problem ganz grundsätzlicher Perspektiviertheiten lenken. Denn das 〈Aufführen〉 unhinterfragt übernommener Inhalte - hier der perspektivischen Darstellung als symbolische Form ohne kontextsensible, kontextkritische Einbettung - ist gängige Praxis in Anleitungen zum Zeichnen, wie im Folgenden anhand zweier TutorialVideos exemplarisch angedeutet wird (siehe unten: Perspektive als Symbolische Form; Perspektive als zeichnerische Leistung; vgl. Bader 2022 i. V;; Karl 2020). Im Vergleich dazu werden im dritten Beispiel mediale Übersetzungsprozesse als solche explizit genutzt, um ein besseres Verständnis für die zugrunde liegenden Prinzipien eines perspektivischen Sehens und Zeichnens anzustossen und so zu einer Ermächtigung der Rezipierenden beizutragen (siehe unten: Perspektive als situierter und situierender Sehvorgang).

\section{Zwischen ästhetisch-explorativer und distanziert- kritischer Analyse}

Mit der Überzeugung, dass ein bildungstheoretisch angestrebtes, medienkritisch geschärftes Bewusstsein insbesondere mit einem erfahrungsbasierten und reflektierenden Registrieren von medialen sowie materiellen Zusammenhängen einhergeht, folgen wir zunächst einer rezeptionsästhetischen sowie repräsentationskritischen Methodik, wie sie im Anschluss an die theoretischen Überlegungen von Manuel Zahn (2020) naheliegt. Zahn entwirft bereits ästhetische Praxis als potenziell kritische Praxis. Kritik meint dabei nicht mehr primär einen distanzierten, selbstreflexiven, begrifflich-rationalen Zugang zu (digitalen) Medien (vgl. ebd., 213). Vielmehr liegt der Akzent darauf, medial zu (inter-)agieren, sich zu involvieren und eigentätig Möglichkeiten und intersubjektive Grenzen zu erkunden. Während Zahn in aktueller Medienpraxis bereits dieses Potenzial am Werk sieht, wenn involvierend mit «Nähe, Immersion, Vernetzung, Serialität, Kooperation und Kollaboration [gearbeitet] und nicht länger auf Distanzierung, Vereinzelung und individuelles kognitives Verstehen» gesetzt wird (ebd., 216), stellt sich für uns die Frage, inwiefern sich eine solche 
Medienpraxis in Tutorial-Videos selbst oder/und in einem ästhetisch-kritischen Umgang mit diesen zeigt oder zeigen kann. Zahn zufolge ist eine medienkritische Praxis erkenntnisstiftend, wenn sie sich nicht als Form von Ablehnung oder Verneinung, sondern als selbstbewusste Erweiterung verstehen lässt:

«Eine kritische Praxis in Relation zu technologisch, politisch, kulturell geregelten und normalisierten Erkenntnisweisen kann demnach nur unter der Bedingung gelingen, dass sie in diesen Erkenntnisweisen verankert - also auch in Teilen affirmativ - ist und zugleich das Ziel verfolgt, über dieselben hinauszugehen. Kritik sucht, Gewissheiten und Ordnungen zu hinterfragen, auf die sie selbst in diesem Akt zurückgreifen muss» (ebd., 217).

Wir versuchen, dieses Postulat in die Praxis umzusetzen, indem wir den Blick nicht nur auf vorhandenes Material richten (drei Tutorial-Videos), sondern auch in Reaktion darauf, ein eigenes praktisches Anschauungsmaterial herzustellen, mit dem sich visuell argumentieren lässt. Der TextBild-Beitrag gelingt, wenn wir zwischen einer ästhetischen Praxis als Kritik und einem distanzierenden, analysierenden, auch reflexiven Vorgehen auf begrifflicher Ebene changieren. Während Zahn zur Veranschaulichung elaborierte künstlerische Praktiken als Beispiele heranzieht (z. B. Trecartin und Fitch 2009-10, «The Re'Search (Re'Search Wait'S)»), dienen zwar auch uns Kunstreferenzen als Inspiration (z. B. zusammengesetzte Polaroids oder Foto-Collagen: Hockney 1985, "Paint Trolley, L.A.»), doch setzen wir mit dem Fokus auf Tutorial-Videos zugleich auf einer basalen, alltagsnahen Ebene medialer Phänomene an, um deren kritische Potenziale aufzuspüren.

Unsere visuelle Argumentationsform ergibt sich aus aktuell weiterentwickelten Methodiken kunstpädagogischer Forschungen, die präzise an Einzelfällen ausdifferenziert wurden und sich dabei sowohl auf $\mathrm{Me}$ thoden der qualitativen Empirie stützen als auch sich bildtheoretischer Zusammenhänge vergewissern (vgl. Sabisch 2019; Bader 2019; Loemke 2019; Karl 2021; Johns 2021; Böhme 2018). Unser Vorgehen ist durch phänomenologische Metareflexion geprägt und orientiert sich ebenso an kunstwissenschaftlichen Herangehensweisen unter Berücksichtigung 
produktions- und rezeptionsästhetischer Aspekte. Dadurch erhalten Bilder in der Verschränkung von Text- und Bildebene konkret den Status von Argumenten. Indem sie bildlogische Operationen eröffnen oder reflektieren, sind sie mehr als Dokumentationen bzw. empirische Quellen.

Wir werden mit Bildern argumentieren, indem wir sie als «visuelles Kontrastmittel» (Schnurr 2015) einsetzen, mit vergleichendem Sehen und mit «Gegenhorizonten in Bildform» (Karl 2014, 2021) validierend arbeiten sowie Formen inhaltlicher Verdichtung und Selektion eines zeichnerischen Visualisierens nutzen (Bader 2019 u. 2020). Diese heterogenen, an den (medialen) Spezifika unseres Forschungsgegenstands orientierten Bildformen eröffnen ein methodisches Spektrum im Umgang mit medialen Bedingtheiten und Übersetzungen, die in unserer Alltagswahrnehmung umso mehr übersehen werden, je technisch einwandfreier sie ablaufen. Die konkrete Materialität (etwa des glatten und leuchtenden Bildschirms, einer Seite DIN A4 im Abendlicht) und Medialität (etwa Schnitt und Kameraführung im Video, Layout eines Textes) tritt zugunsten der konsumierten Inhalte oft zurück. Bei der Wahrnehmungssensibilisierung für unseren Untersuchungsgegenstand kommen deshalb genau solche oft unbemerkten Dimensionen der Materialität und Medialität immer wieder bewusst zum Einsatz. Mit unserem fallspezifisch entwickelten Anschauungsmaterial fokussieren wir im Kapitel «Perspektiven und Perspektivierungen» vor allem auf Räumlichkeit, während im Kapitel «ästhetische Erkundungen und Erfahrungen» vor allem Zeitlichkeit als Grundbedingung von Wahrnehmung bedeutsam wird.

Ferner gehen wir im Zuge visueller Argumentationslogiken von der Metareflexivität des Bildhaften aus, da ein Bild (oder eine Perspektive) nicht nur etwas, sondern zugleich das eigene Bildsein (oder Perspektiviertsein) zeigt. Diese medientheoretische Dimension, wie sie Mersch mit dem Begriff der «Zerzeigung» immer wieder ins Bewusstsein holt (Mersch 2014), ermöglicht uns, in den Analysen zugleich sehr konkret und sehr allgemein zu sein. Dabei sollen auch themenübergreifende implizite Erwartungen an die medialen Formate von Erklär- und Tutorial-Videos (vgl. Dogerloh und Wolf 2020) sichtbar werden. 


\section{Perspektiven und Perspektivierungen}

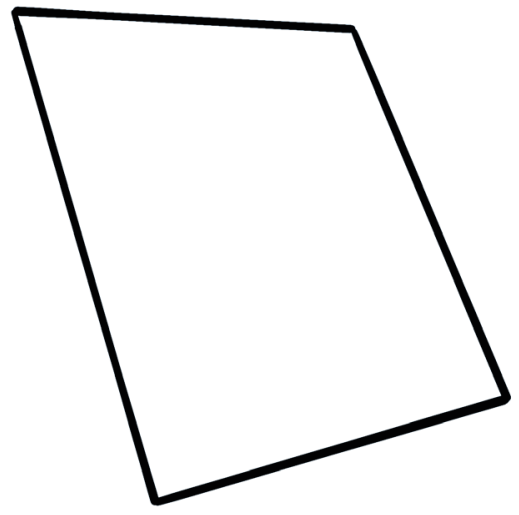

Abb. 3: Verzerrtes Rechteck (Zeichnung: Nadia Bader).

Um im Folgenden der Perspektive als symbolischer Form differenzierter auf den Grund zu gehen, lohnt eine Vergegenwärtigung der «Perspektiviertheit der Perspektive». Schon zu Beginn der Erfindung der Perspektive hantierten die damaligen Expertinnen und Experten mit zusätzlichen Instrumenten, Apparaturen und Sehvorrichtungen, um eine räumliche Übersetzung zu 〈sehen〉 und als angemessen zu erfahren. Diese visuelle Behauptung konnten sie mit Methoden der Geometrie beweisen. Dass es dabei implizite Prämissen gibt, wie sich Einzelne vor dem zweidimensionalen Artefakt platzieren müssen, zeigen nicht zuletzt die Abbildungen dieser Apparate selbst. Man denke nur an Albrecht Dürers Holzschnitt «Der Zeichner der Laute» (1525) mit entsprechender Konstruktionsanordnung zum Zeichnen von Perspektive.

Wer sich nun zur Bewusstwerdung dieser Prämissen so vor dem Bildschirm de-platziert, dass das gezeichnete Rechteck (Abb. 3) nicht mehr verzerrt erscheint, vermag die Linearperspektive gegen deren Regeln zu wenden und Zusammenhänge sichtbar werden zu lassen - nicht zuletzt jene körperlichen, sich selbst gerade mit einem befremdlich schief vor die Nase gehaltenen Bildschirm von Laptop oder IPad zu ertappen. Erfahrbar 
wird also die Vorannahme der Zentralperspektive, Bilder seien fensterartige Durchblicke, auf deren virtueller Glasscheibe als Bildebene sich die visuellen Daten quasi 〈eintragen〉 lassen. Doch schon allein der 〈schiefe〉 Blick auf den Computerbildschirm zeigt, dass diese Ebenen in den seltensten Fällen so ineinanderfallen, oder dass wir davor einäugig und unbeweglich werden. Jede Bewegung des Standpunktes würde die bisherigen visuellen Daten in dieser Darstellungslogik zu〈Fehlern〉 machen. Anhand dieses kleinen Experiments wird deutlich, was in Tutorial-Videos zum Thema des perspektivischen Zeichnens zumeist verdeckt bleibt: der Widerspruch zwischen im Moment gesehener und gezeichneter Perspektive. Die provokative Aufforderung, die Betrachtende dazu anregt, einen systemexternen Standpunkt einzunehmen, macht bewusst, wie sehr das perspektivische Display uns als Betrachtende körperlich ausrichtet, zentriert und stillstellt. Das Hinein- und Hinaustreten in den virtuellen Bildraum als implizite Erfahrung von Einpassen und Verzerren wird so wahrnehmbar und explizit.

Die Thematisierung der Perspektive sei so bereits in ihrer doppeldeutigen Beispielfunktion ins Spiel gebracht, als Gegenstand der TutorialVideos und zugleich als wirksames Dispositiv jeder Kameraaufzeichnung. Hier unterscheiden sich in der Regel die perspektivischen Inblicknahmen von Tutorin oder Tutor, Rezipierenden und Kamera, meist ohne dies zu problematisieren. Eine stillgestellte Kamera verweist auf das oft ignorierte Kernproblem, nämlich dass Perspektive nicht als solche (gesetzmässige, regelgeleitete Darstellungsform) präexistiert, wie dies die Zeichnung vorgibt, sondern, dass sie erst im Moment des Blickens immer neu und situationsabhängig erzeugt wird. In der Regel bemerken wir nicht, wie viele Vorerfahrungen in unser Sehen bereits eingelagert sind (vgl. Hoffmann 2015). Wenn es folglich nicht nur um das 〈Auflesen〉 von Zeichen gehen soll, müssten diese Vorerfahrungen zuallererst ins Bewusstsein geholt werden: hier in Form einer Aufforderung, sich auf die notwendige körperliche Mühe einzulassen, um die (eigene) Ausrichtung zu durchbrechen und so das besagte Rechteck zu sehen. Derartige Dezentrierungen des Subjekts tauchen bereits in der Geschichte der Kunst unter dem Begriff «Anamorphose» seit der Renaissance auf, jener Epoche, die für die Erfindung der zentralperspektivischen Konstruktion steht. 


\subsection{Perspektive als symbolische Form}

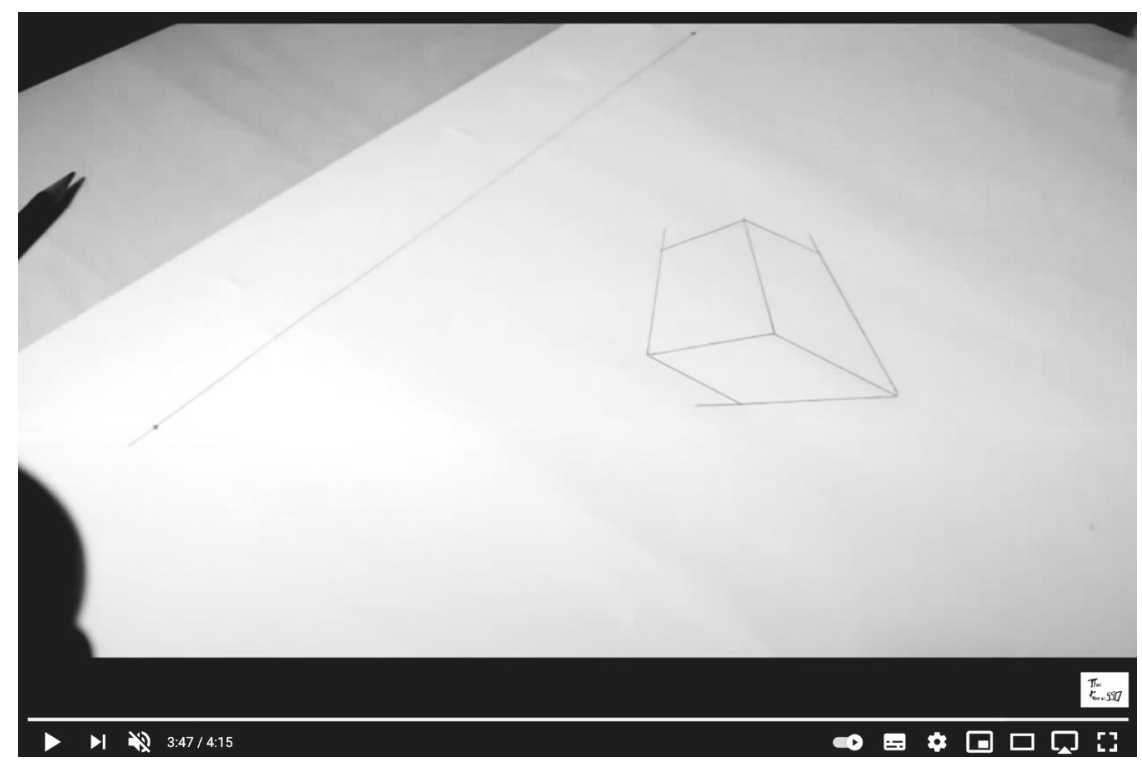

Abb. 4: «Tutorial Zeichnen lernen - 2 Fluchtpunkt Perspektive», Brian thekone99o, 2014, URL: https://www.youtube.com/watch?v=jswknMPx308 (bearbeitetes Videostandbild: Min. 03:47, Nadia Bader).

Im ersten Beispiel, dem Tutorial-Video von Brian thekoneggo (2014) mit dem Titel «Tutorial Zeichnen lernen - 2 Fluchtpunkt Perspektive», wird während ca. 4 Minuten vorgeführt, wie auf einem Blatt Papier mithilfe von Lineal und Bleistift ein Kubus gezeichnet werden kann, der räumlich wirkt, weil seine Kanten auf zwei Fluchtpunkte hin ausgerichtet sind. Brian zeigt und erklärt Schritt für Schritt, wie dabei vorzugehen ist - angefangen beim Zeichnen der parallelen Horizontlinie nahe der oberen Blattkante, über das Definieren zweier Fluchtpunkte auf dieser Linie innerhalb des Blattraumes links und rechts, bis hin zum Zeichnen des Kubus, dessen vertikale Kanten senkrecht und dessen Seiten auf die zwei Fluchtpunkte hin ausgerichtet gezeichnet werden. Das Videostandbild (Abb. 4) zeigt, dass die Kamera dabei etwas erhöht von links auf die Arbeitsfläche ausgerichtet ist, wobei das Blatt Papier unten und rechts angeschnitten aufgezeichnet wird. Dieser Aufnahmewinkel ist der Perspektive einer Sitznachbarin bzw. 
eines Sitznachbarn ähnlich, die bzw. der dem Zeichner von der Seite her bei der Arbeit (womöglich privat zu Hause) zuschaut, sodass sich hier insbesondere Peers angesprochen fühlen können.

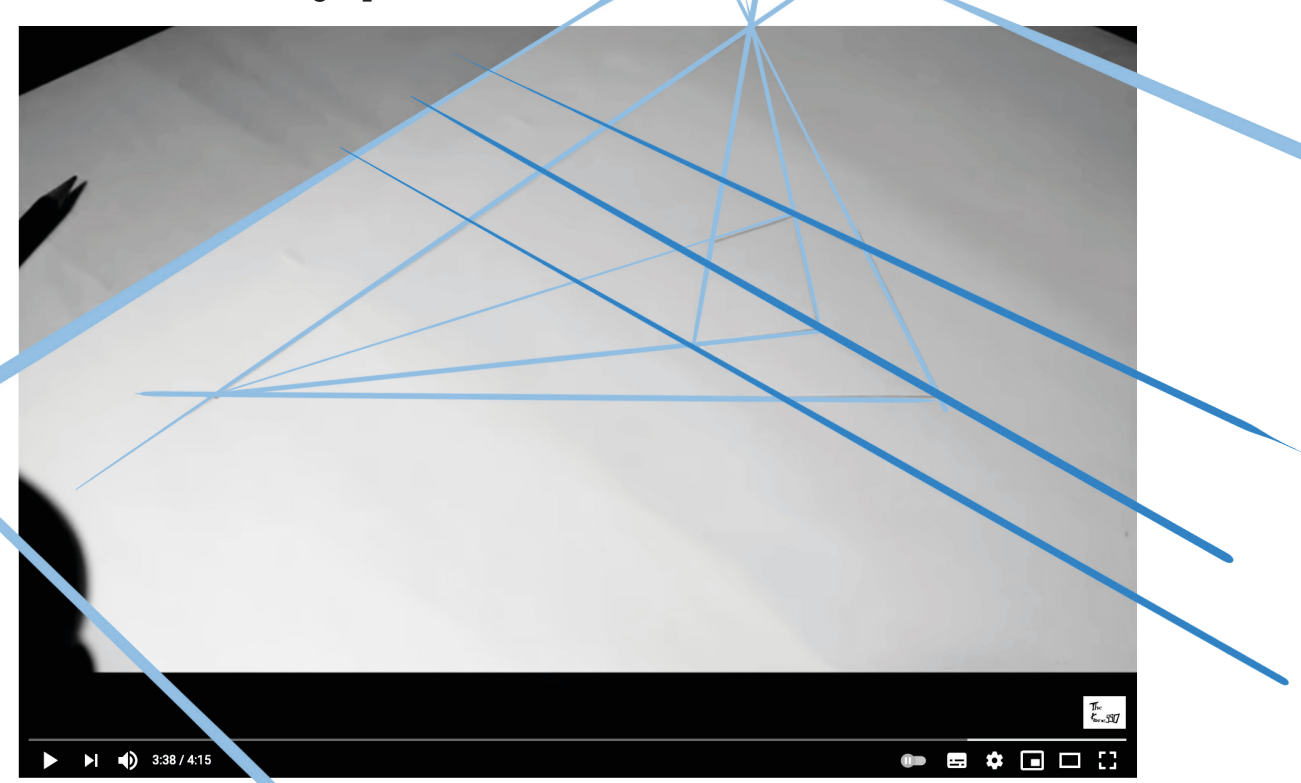

Abb. 5: Visualisierung zu Abbildung 4 (bearbeitetes Videostandbild: Nadia Bader).

Bemerkenswert ist daran zunächst, dass wir als Betrachtende somit nicht die Perspektive des Zeichners einnehmen können und auf eine seitlich schräge Ansicht der Zeichnung verwiesen sind. Einerseits ist zwar nachvollziehbar, dass und wie die seitlichen Kanten des gezeichneten $\mathrm{Ku}$ bus auf die Fluchtpunkte hin ausgerichtet werden. Andererseits ist aber die tatsächliche räumliche Wirkung der Zeichnung nicht überprüfbar. Beispielsweise lässt sich nicht erkennen, ob die senkrechten Kanten des Kubus tatsächlich senkrecht gezeichnet wurden, da wir diese aus unserer Perspektive nur als auf dem Blatt 〈liegend〉 wahrnehmen können (Abb. 5). Umgekehrt wird die Raumillusion erst vom 〈richtigen〉 Betrachtendenstandpunkt aus gesehen wirksam und bleibt uns hier aufgrund der Kameraeinstellung verwehrt. 


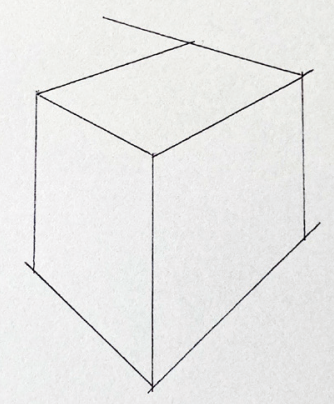

Abb. 6: Zeichnung nach Anleitung (Notburga Karl).

In einem nächsten Schritt ist daher methodisch aufschlussreich - im Sinne der Produktionsästhetik -, selbst eine Zeichnung gemäss der Anleitung des Tutorial-Videos anzufertigen (Abb. 6). Mit Blick auf die Zeichnung fällt auf, dass die unteren seitlichen Kanten des Kubus in einem relativ spitzen Winkel zusammenlaufen. Im Abgleich mit alltäglichen Seherfahrungen drängen sich womöglich Fragen auf: Wirkt der Kubus vielleicht etwas verzerrt oder würde er wirklich so aussehen? Aus welcher (imaginären) Betrachtendenposition blicken wir eigentlich auf den virtuellen, dreidimensionalen Körper? Wie nah oder fern ist der Kubus, wenn er uns in dieser Ansicht erscheint?

In der folgenden Bildserie nähern wir uns im Sinne einer visuellen Argumentation - hier als visueller Vergleich und als Gegenhypothese in Bildform (s. o.) - an die im Tutorial-Video gezeigte und die nach Anleitung erzeugte Zeichnung an. Mit dem Handy wird zunächst die selbst angefertigte Zeichnung (Abb. 7 bis 9) und dann ein tatsächlicher Acrylglaswürfel (Abb. 10 bis 12) aus verschiedenen Perspektiven abfotografiert, um das einäugige Blicken zu simulieren. 

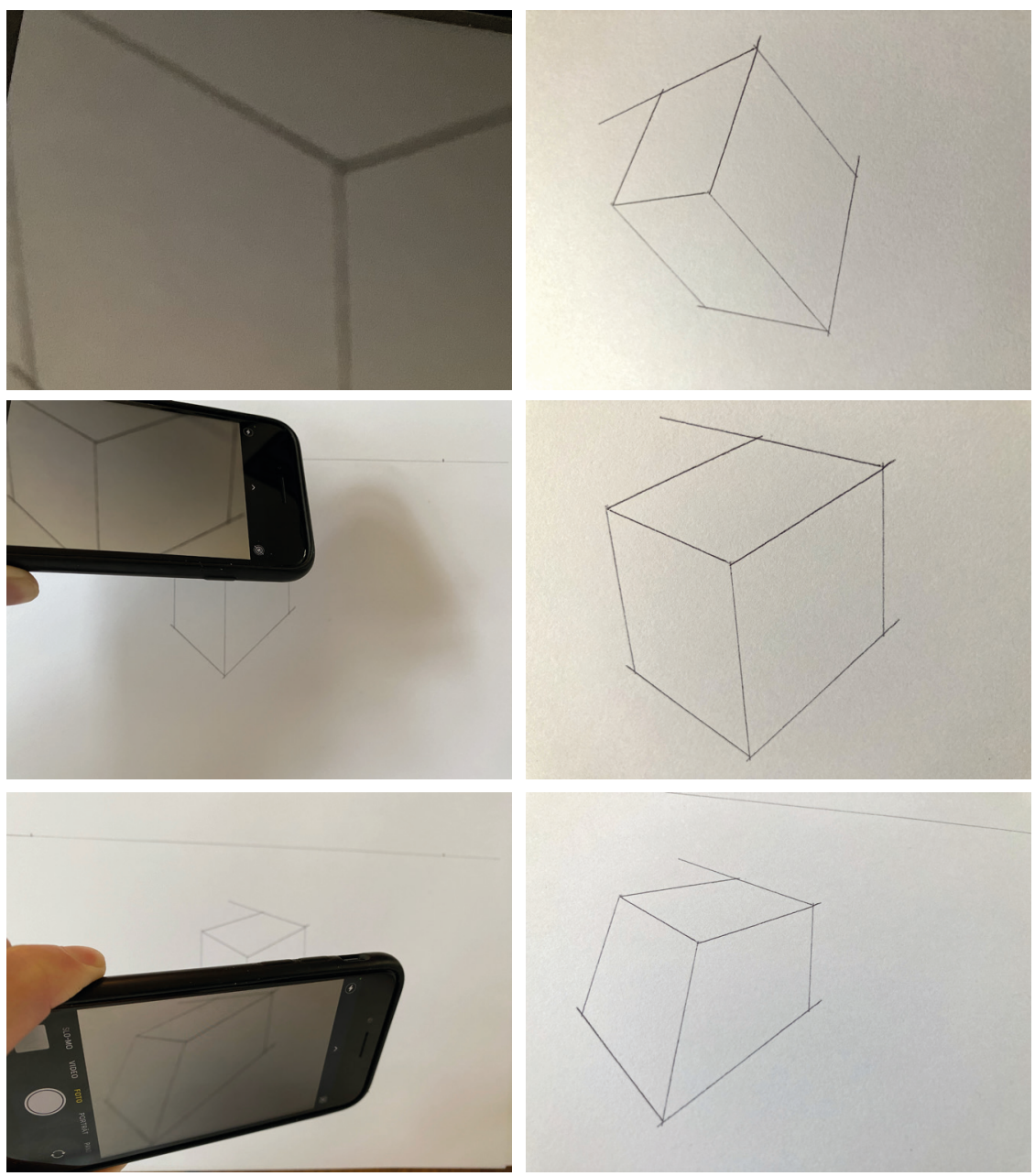

Abb. 7a, 7b, 8a, 8b, 9a, 9b: Fotografische Situationen und fotografierte Zeichnungen (Fotos: Notburga Karl). 

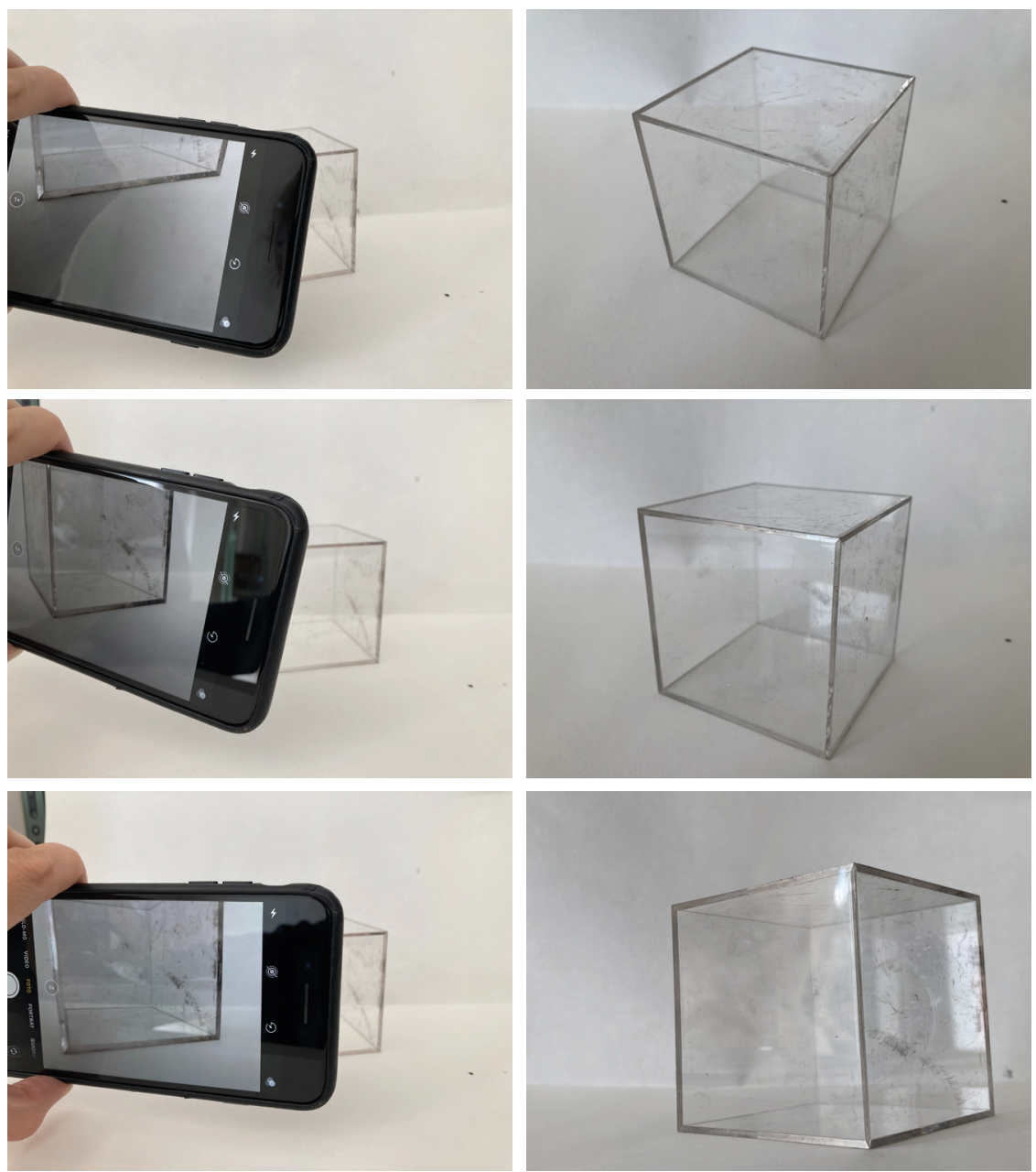

Abb. 10a, 10b, 11a, 11b, 12a, 12b: Fotografische Situationen und fotografierter Acrylglaswürfel (Fotos: Notburga Karl). 


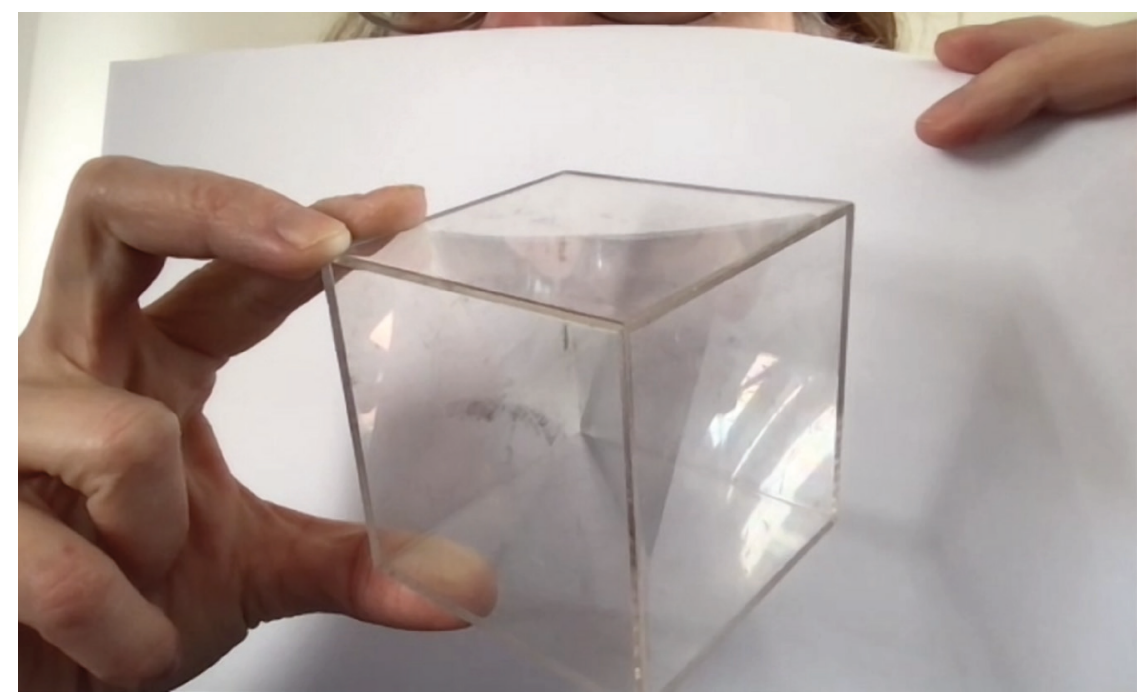

Abb. 13: PPP, Tagungslabor: Performen perspektivischer Ansichten eines Acrylglaswürfels via ZOOM (Bildschirmfoto: Notburga Karl und Nadia Bader).

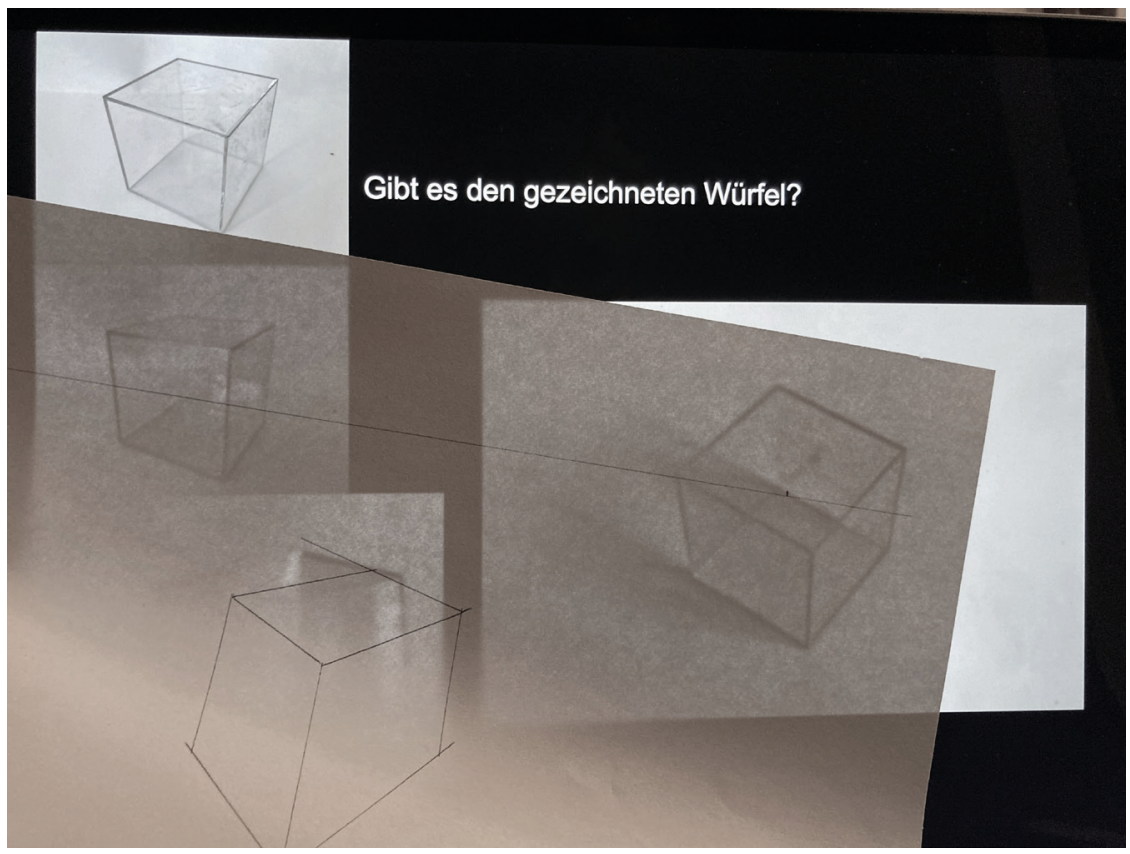

Abb. 14: PPP, Tagungslabor: Gibt es den gezeichneten Würfel? (Foto: Notburga Karl). 
Die Gegenüberstellung der Aufnahmesituationen und der so entstandenen Fotografien zeigen teilweise subtile und teilweise markantere Verschiebungen. In der ersten Bildserie wirkt die Zeichnung mal ver- und mal entzerrter. Anhand der zweiten Bildserie lässt sich der Versuch erahnen, die Ansicht des gezeichneten Kubus am dreidimensionalen Modell nachzustellen, was jedoch nicht so ganz zu gelingen scheint (Abb. 14). In der fotografierten Ansicht des Acrylglaswürfels, die der Zeichnung noch am nächsten zu kommen scheint (Abb. 10b), sind die vertikalen Kanten eben gerade nicht senkrecht, sondern streben auf einen dritten Fluchtpunkt zu, der sich unten, ausserhalb des Bildraums befindet.

Der Einsatz des Handys wirkt hier distanzierend, steht jedoch zugleich für unmittelbare, mediale Verfügbarkeit und eine Art externes, ausgelagertes Auge (vgl. Selfie Stick), welches den Blick vom fotografierenden Subjekt löst und dezentriert. Dadurch wird der Betrachter visuell vom Bild, von der Perspektive des gezeichneten Würfels (entkoppelt): Auf dem Bild, das wir jetzt zeigen, findet erneut eine Verortung statt - weil die Hände und die Geste des Zeigens sichtbar sind (oben: Schräghalten des Displays, unten: Hochhalten/Schweben lassen des Würfels). In der Bildserie werden einerseits im simultanen Überblick und Nebeneinander der Fotografien verschiedene Perspektiven und Facetten desselben Gegenstandes und derselben Situation sichtbar. Werden die Fotografien andererseits als Bildsequenz betrachtet (von links nach rechts und von oben nach unten), suggerieren sie ein Nacheinander und eine Blickbewegung. Durch diese Bewegung beim Sehen wird die Zeichnung gewissermassen zu einem 〈gemorphten> Resultat verschiedener Einzelansichten. Ein filmisches Sehen einer Bildsequenz wird so zu einem Übergangssehen. In Abbildung 14 wird darüber hinaus die Medialität des Bildträgers durch das Überlagern und Durchscheinen des beleuchteten Displays durch das Zeichenpapier hindurch thematisiert.

Indem das Zeigen selbst gezeigt wird, wird es mitreflektiert. Die zwei Bildserien können so als Visualisierung dessen gelten, was Repräsentationskritik meint: Das Blicken selbst - hier veranschaulicht durch den Blick der Kamera - ist bereits ein Vorgang des Verschiebens, wobei es keine ursprüngliche, originäre Blickweise bzw. Ansicht gibt. Zugleich wird die zeichnerische Darstellung, wie sie im Tutorial-Video vorgeführt und 
in der eigenen Zeichnung nachgeahmt wird, als Konstruktion und Fiktion erkennbar: Ein physisches Nachstellen der Ansicht des Kubus, wie sie in der zeichnerischen Darstellung präsentiert wird, kann nicht gelingen. Perspektivisches Sehen und Darstellen erweist sich hier als abhängig von spezifischen zeiträumlichen Situationen, was der Vorstellung von einem allgemeingültigen System widerspricht bzw. die Spezifizität der perspektivischen Darstellung (mit einem, zwei oder drei Fluchtpunkten) deutlich macht: Sie ist ein Spezialfall, nicht der Normalfall (vgl. Asisi 2020).

\subsection{Perspektive als zeichnerische Leistung}

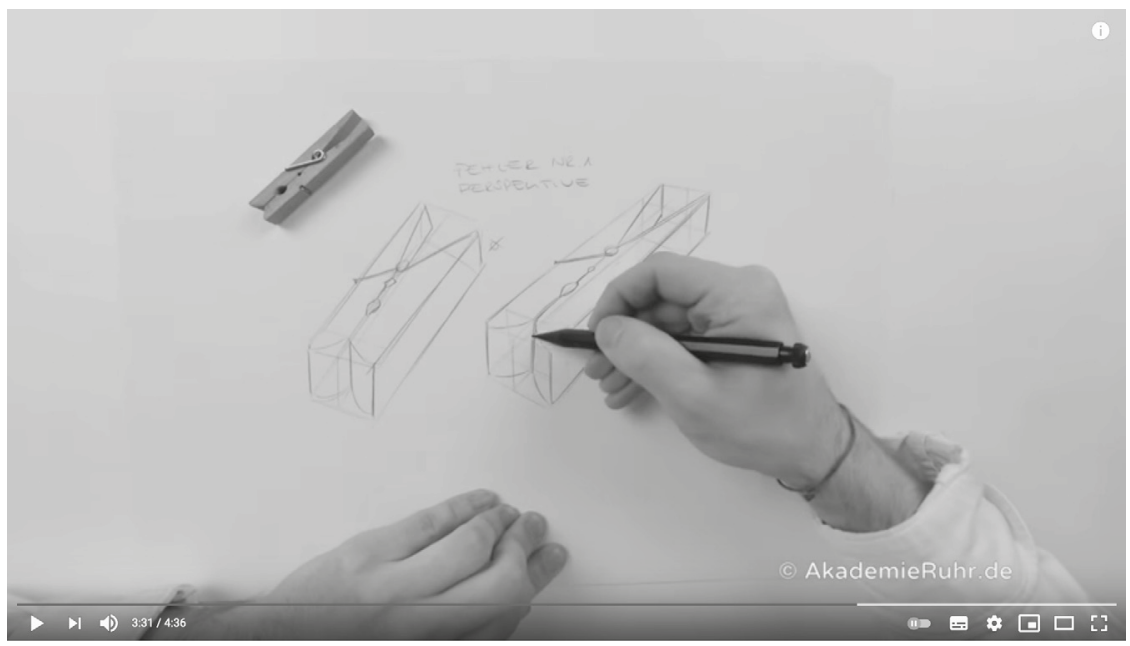

Abb. 15: «TOP 5 Fehler beim Zeichnen. Ein Muss für jeden, der richtig zeichnen lernen möchte!» (AkademieRuhr 2017; https://www.youtube.com/ watch?v=rZOczWwByvM, ${ }_{\text {i }}$ bearbeitetes Videostandbild: Min. 03:31; Nadia Bader).

Das zweite Tutorial-Video, das uns hier als Beispiel dient, ist das erste Video einer kurzen Reihe zum Thema «TOP 5 Fehler beim Zeichnen. Ein Muss für jeden, der richtig zeichnen lernen möchte!» (AkademieRuhr 2017), welches sich mit Hinweisen zur (richtigen/falschen) perspektivischen Darstellung beschäftigt. Mit Blick auf das Videostandbild (Abb. 15) zeigt sich, dass wir diesmal als Rezipierende beinahe senkrecht von oben auf eine 
Arbeitsfläche blicken, auf welcher oben links eine kleine, hölzerne Wäscheklammer und im Zentrum ein Blatt Papier mit zwei gezeichneten Wäscheklammern liegt.

Im Video erfahren wir, dass die Zeichnung links als Negativbeispiel fungiert und die Zeichnung rechts eine gelungene perspektivische Darstellung zeigen soll. In unserem Zusammenhang scheint hier der Vergleich zwischen der auf dem Tisch liegenden Klamnep und der gelungenen Zeichnung interessant: Während wir von oben auf die daliegende Klammer blicken, zeigt sich in der Zeichnung eine Ansicht, die deutlich in die Tiefe des (virtuellen) Blattraums hinein strebt (Abb. 16).

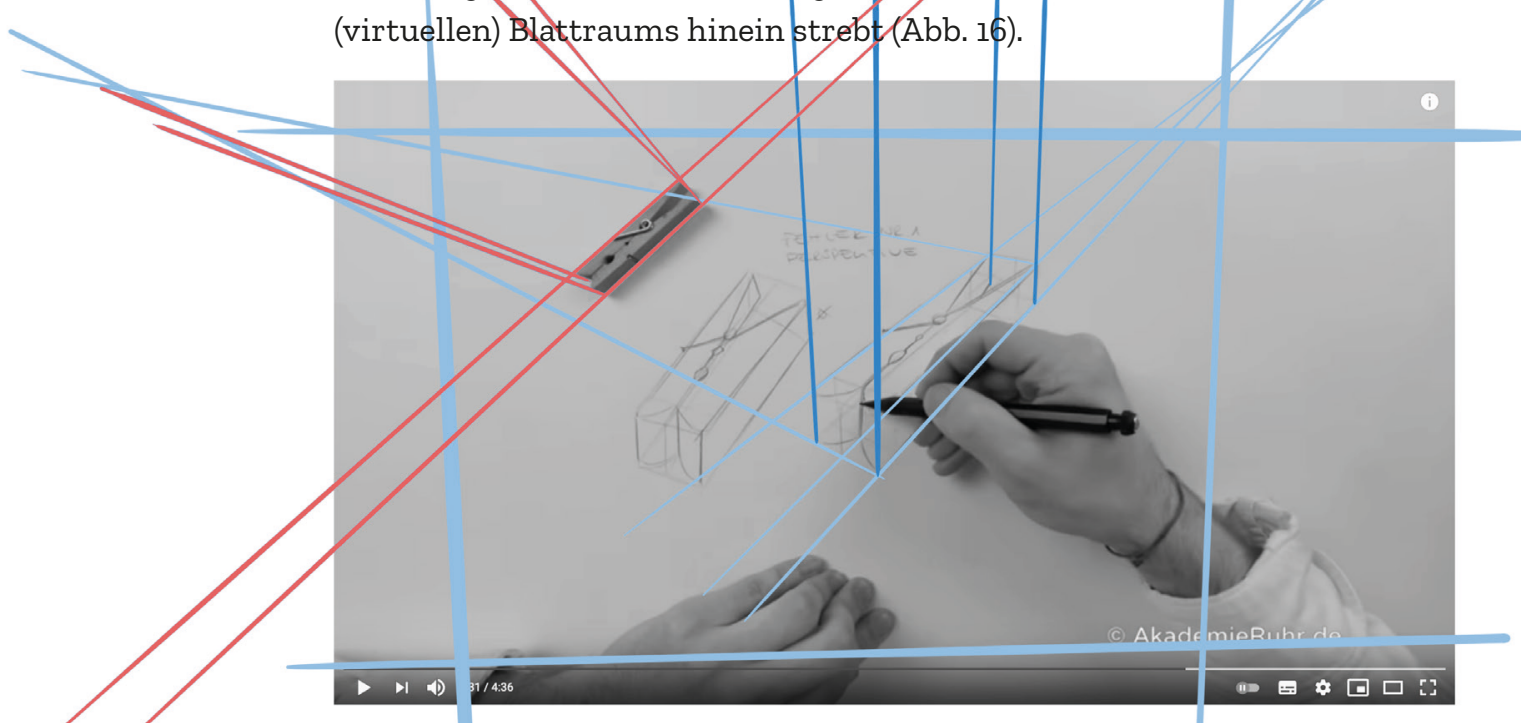

Abb. 16: Visualisierung zu Abbildung 15 (bearbeitetes Videostandbild: Nadia Bader).

Der Vergleich legt zunächst nahe, dass die Klammer aus der Perspektive des Zeichners, der am Arbeitstisch sitzt, eine andere Ansicht bietet. Als Rezipierende des Videos können wir zwar nun die Zeichnung weitgehend unverzerrt vor uns sehen, doch bleibt uns auch hier die Perspektive des Zeichners verborgen, wodurch kein Vergleich zwischen der tatsächlichen Ansicht des Gegenstandes und dessen Darstellung möglich ist. Bei näherer Betrachtung lässt sich allerdings vermuten, dass sich der Zeichner nur bedingt an der Ansicht des vor ihm liegenden Gegenstandes orientiert und diesen stattdessen in seiner Zeichnung perspektivisch de- und 
re-konstruiert. Die kleine Wäscheklammer aus dem im Video ersichtlichen Abstand zu betrachten, würde kaum eine so starke perspektivische Verjüngung der Längskanten ergeben. Wir haben es also eher mit einer übersteigerten perspektivischen Darstellung zu tun, die den Effekt der Raumillusion besonders betont.

Die Kameraeinstellung, die gleichmässige Ausleuchtung der Arbeitsfläche, der besondere Zeichenstift und die effektvolle Qualität des positiven Beispiels zur perspektivischen Darstellung der Wäscheklammer vermitteln einen professionellen Anspruch. Im Kontrast zu einer eher informell anmutenden Ansprache im Video wirkt dieser, insbesondere visuell vermittelte Anspruch distanzierend: Als Rezipierende erhalten wir nur einen selektiven Einblick und sind so insgeheim darauf angewiesen, dem Profi, der uns zeigt, wie man eine perspektivisch wirkungsvolle Zeichnung erstellt, in seinem Wissensvorsprung zu vertrauen und in seiner Vorgehensweise bestmöglich Schritt für Schritt $\mathrm{zu}$ folgen. Was die voraussetzende Wahrnehmung angeht, ist durch die zwei unterschiedlichen Standpunkte qua Perspektiveinstellung im Video eine Perspektivübernahme oder ein vertieftes Verstehen der perspektivischen Darstellungslogik nur bedingt möglich. Vielmehr wird eine Übernahme von regelgeleiteten Techniken und Tricks angeregt, in die der Profi seine Klientel einweiht (vgl. Modzelewski 2021: «100 geniale Zeichentricks»). Rezipierende werden dadurch eher als Laien denn als professionelle, eigenständig Lernende adressiert. Zugleich wird der unspezifische Ort des Zeigens als Ort eines allgemeingültigen Wissens inszeniert, dem sich der Zeichner durch das Weglassen jeglicher Störung (vgl. weisser Pullover) selbst unterwirft. Medienästhetische Mittel werden hier in wirkungsvoller Weise eingesetzt, bleiben jedoch implizit. Es bleibt fraglich, ob die performativ-flüssige Darstellung der eigenen zeichnerischen Kompetenz das einlöst, was sie verspricht: dass die Rezipierenden danach selbst in der Lage sind, auch andere Dinge als Wäscheklammern perspektivisch zeichnen zu können (vgl. detailliertere Analyse: Bader 2022/i. V.).

In beiden Tutorial-Videos soll den Rezipierenden das perspektivische Zeichnen erklärt werden. Im ersten Beispiel werden konkrete zeichnerische Handlungen und im zweiten Beispiel eine wirkungsvolle perspektivische Darstellung vorgeführt, die zur Nachahmung anstiften sollen. Bei der 
Betrachtung der Videostandbilder (Abb. 4 und Abb. 15) fallen mediale sowie inhaltliche Brüche auf: In beiden Videos bleibt den Rezipierenden die Perspektive der Zeichnenden - auf die Zeichnung, auf den Gegenstand - und damit eine eigene Überprüfung des Sichtbaren und somit ein wesentlicher Aspekt im Hinblick auf einen Nachvollzug des Gezeigten verwehrt. Im Zusammenhang mit dem Thema Perspektive kann diese Unterschlagung als besonders bedeutsam gelten und verweist auf eine mediale (sowie zeichendidaktische) Verkürzung, die einem vertieften Verstehen insgeheim sogar entgegenwirkt: Rezipierende können zwar das gezeigte Vorgehen Schritt für Schritt (Beispiel 1) oder die wirkungsvolle Darstellung (Beispiel 2) nachahmen. Zugleich bleibt aber völlig unklar, weshalb so (und nicht anders) vorzugehen ist, woran sich die Zeichner tatsächlich implizit orientieren und was dies für die Wirkung der Zeichnung bedeutet bzw. weshalb gerade diese Darstellungsweise die ideale Lösung sein soll. Die (gut gemeinten) Tipps bleiben weitgehend kontextfrei und umgehen so die Kernfrage eines kompetenten Umgangs mit Bildern: inwiefern Darstellungsabsicht und Wirkung des gestalteten Bildes korrespondieren. Dies hat Konsequenzen für die Bildungspotenziale solcher Videos:

«Wird im Unterricht [oder in Tutorial-Videos] die bildsprachliche Kompetenz auf die operative Seite begrenzt, wird dem Bildsprachenerwerb sein grundsätzlicher Sinn entzogen: seine kommunikative Funktion.» (Marr 2015, 17)

Wir haben es also mit einer gesteigerten Problematik der Perspektiviertheit - im räumlichen wie symbolischen Sinne - zu tun, die in den Tutorial-Videos implizit machtvoll wirkt, von den Präsentierenden jedoch vermutlich nicht bedacht wird (weder in den Videos noch in den Shownotes wird darauf eingegangen). Dies wirft ganz grundlegende Fragen einer (medialen) Repräsentation auf: Was ist (un)sichtbar? Was wird (nicht) gezeigt? Und welche alternativen Formen der (visuellen) Darstellung sind denkbar? 


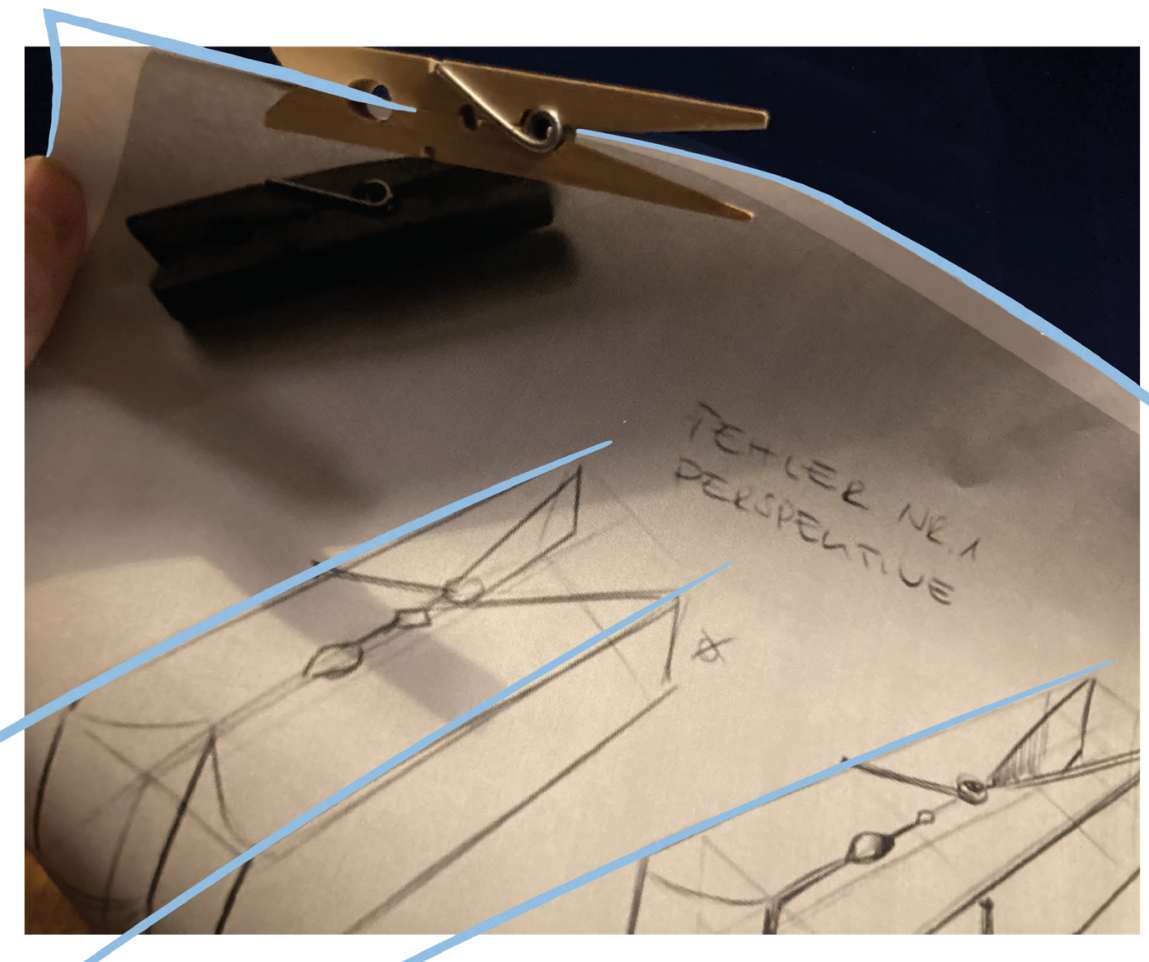

Abb. 17: PPP, Tagungslabor: medial-materielles «Zurückblättern» (Foto: Notburga Karl). 


\section{4. Ästhetische Erkundungen und Erfahrungen}

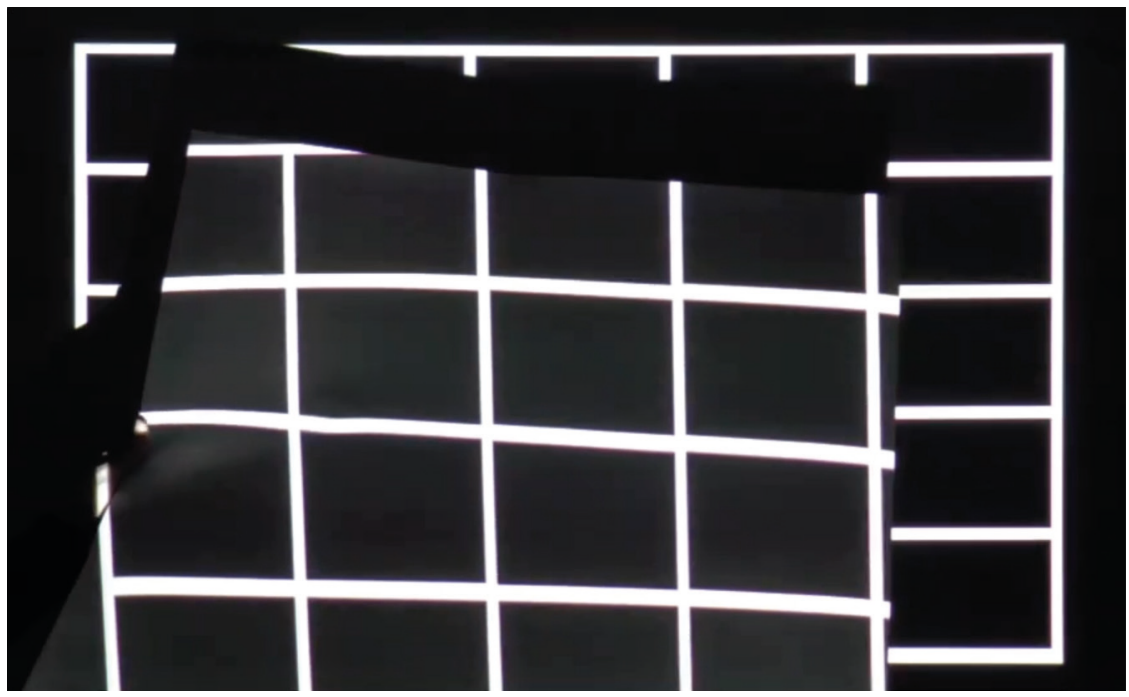

Abb. 18a: PPP, Tagungslabor: «Zoom-Grid» (Videostandbild: Notburga Karl, 2021).

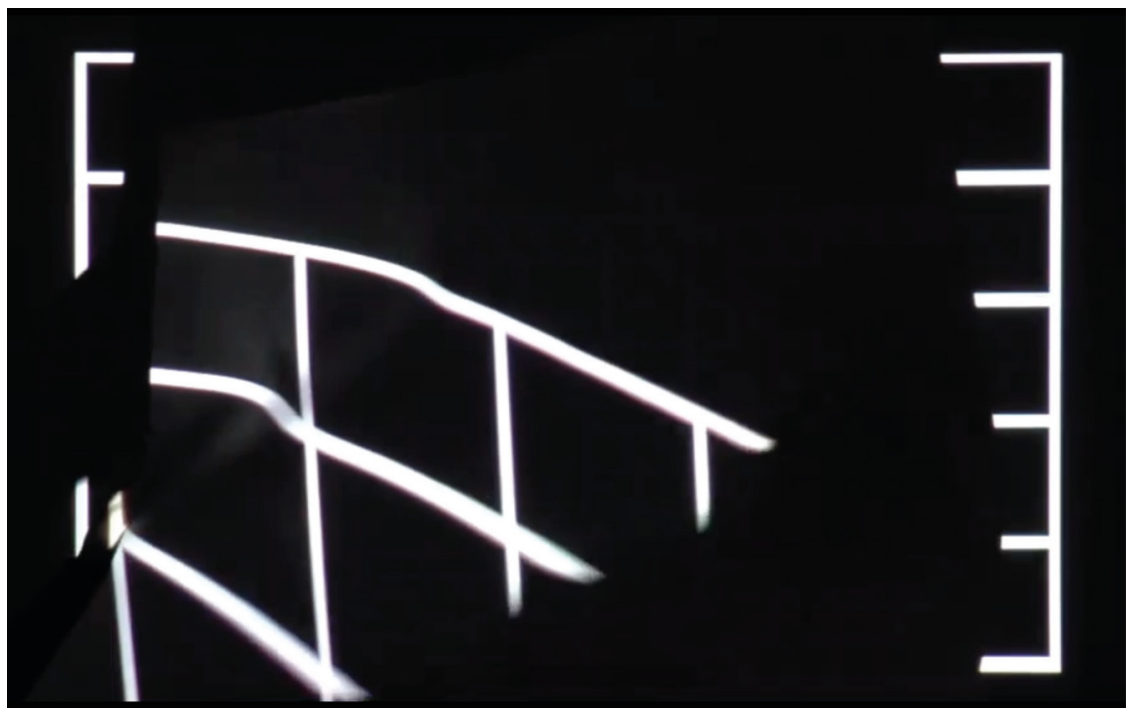

Abb. 18b: PPP, Tagungslabor: «Zoom-Grid»(Videoclip: Notburga Karl, 2021). Der Videoclip ist abrufbar unter: https://www.medienpaed.com/article/ download/1364/1107. 
Das nächste Anschauungsexperiment bzw. die nächste Seherfahrung gibt der Bedeutung der Zeitlichkeit nun mehr Gewicht und schärft damit den Blick für spezifische Zeitspannen in Tutorial-Videos. In der Gegenüberstellung eines Videostandbildes (Abb. 18a) mit der Sequenz des Videoclips (Abb. 18b) wird die Bedeutung der Zeit im Sinne einer Zeitspanne des Andauerns im Sehakt unterstrichen und als Vorgang sinnlichen Erkennens wahrnehmbar.

Die Konstellation ist über das Videostandbild schnell durchschaut: Ein in das Projektionsfeld gehaltenes Papier verzerrt das abgebildete Raster, welches an gesichtslose ZOOM-Fenster in der Galerieansicht mit 25 Kacheln erinnert. Was das exemplarische Videostandbild allerdings verschweigt, ist etwas, was sich im Clip als «Konstellieren» fassen lässt: das unausgesetzte Fliessen der Figuration über den nicht länger planen Bildgrund, der bisweilen dazu selbst in Bewegung gerät. Die konventionelle Bildkonstellation von (bewegter) Figur vor (ruhigem, flächigem) Grund ist invertiert und irritiert, und zwar kontinuierlich. Hat man die Relation erkannt, geschieht sinnliche Erkenntnis erst durch das Prozessieren eines ständigen Übergangs, als kontinuierliches Relationieren, auch wenn es sich dabei immer um dieselben Bildgegenstände handelt. Denn hier wirken im Sinn einer andauernden Zeitspanne Vergangenes und Zukünftiges als nachwirkende, sich (technisch) überlagernde Bilder so ineinander, dass sich der Moment nicht als etwas ausserhalb der Raumzeit Greifbares, sondern als ständig sich entziehendes Übergangsmoment während des Betrachtens fassen lässt.

Analog zum Unterschied zwischen einem theoretischen Wissen über Perspektive und dem perspektivischen Sehen als Wahrnehmungspraxis wird hier ein Wesensunterschied deutlich, der im medialen Stillstellen zum repräsentativen Bild (Kamerabild sowie Zeichnung) verflacht. Dieses Problem haftet ebenfalls den Tutorial-Videos insofern an, als dass sie deduktiv oft von einem vorgefertigten Wissen über Perspektive ausgehen und dann davon das Zeichnen abhängig machen. Das heisst, auch die Kameraeinstellung auf die Wahrnehmungs- und Zeichensituation ist stillgestellt und arbeitet mit einem vorgegebenen fixen Betrachendenstandpunkt. Stattdessen könnte induktiv gerade mithilfe einer beweglichen Kamera der Sehvorgang unter den Prämissen perspektivischen Sehens 
vorgeführt und analysiert werden, um sich dabei gar auf die Perspektive als Wahrnehmungserfahrung der Rezipierenden einzulassen und von dort zu verallgemeinerbaren Schlüssen zu kommen. Im nächsten Beispiel werden diese Aspekte aufgegriffen.

Solche induktiv vorgehenden und kritischen Potenziale phänomenologisch reflektierter Wahrnehmung sind auf ein vergleichendes Andauern der Wahrnehmung angewiesen. Sobald sich zum Beispiel das 〈Was〉 der Wahrnehmung ins 〈Wie〉 und 〈Wodurch〉 wendet, gelingt das, was Mersch im Sinne der «Zerzeigung» fasst (Mersch 2014). So einen Wahrnehmungsumsprung als Medium ästhetischer Erfahrung stellt Michael Lüthy mit Bezug zu Wittgenstein als Aspektwechsel heraus. Im Umspringen liegt das Erkenntnisstiftende, das sich (je nach Phänomen) durch einen zeitlichen Verlauf erst zeigt (Lüthy 2012). Die Tatsache des Umspringens ist gegenüber dem Vorgang des Umspringens (vgl. Hase-Ente-Bild bei Wittgenstein, in Lüthy 2012, 133) weniger als ein semiotisches Umspringen innerhalb zeichenhafter Bedeutungen, sondern als ein Umspringen von Wahrnehmungseinstellungen relevant. Die Regeln der Perspektive werden also nur als konstruierte erfahrbar, wenn die Kamera das Umspringen der Aspekte und mehr oder weniger einrastenden Ansichten vorführt.

\section{Perspektive als situierter und situierender Sehvorgang}

Das Tutorial-Video «Der Fluchtpunkt beim Zeichnen: Einfach erklärt» von Yadegar Asisi (2020) erscheint uns aufschlussreich, da u. a. mit dieser anderen Zeitlichkeit gearbeitet wird, die mit der Art und Weise des Erklärens und Zeigens verquickt ist. Während der ca. 31 Minuten des Videos (eine deutlich überdurchschnittliche Länge im Vergleich zu anderen Videos zum selben Thema) nähert sich Asisi über (auch medial) verschiedene Zugänge dem Thema Perspektive an. Anstatt also direkt und in Kürze zu zeigen, wie beim Zeichnen mithilfe von Fluchtpunkten vorzugehen ist (Tutorial-Video 1) oder was zu vermeiden ist (Tutorial-Video 2), wird das Thema mehrfach umkreist, bis schliesslich die zeichnerische Konstruktion eines Raums nach den Regeln der Fluchtpunktperspektive vorgenommen wird. 
Asisi scheint darum bemüht zu sein, die Zuschauenden auf einen Weg des Erkundens mitzunehmen und ihnen so eine ästhetische Erfahrung im Sinne eines bewusste(re)n Wahrnehmens der (eigenen) Wahrnehmung zu ermöglichen. Das Umkreisen und die verschiedenen Annäherungsversuche zeigen sich insbesondere in den medial unterschiedlichen Darstellungsweisen. So lässt sich argumentieren, dass in diesem Tutorial-Video mit spezifischen medialen Mitteln ein Wahrnehmungsvorgang am Beispiel der Perspektive vorgeführt wird, wobei mediale Brüche und Wechsel als Vermittlungsstrategien gelten können, welche die (eigene) Perspektiviertheit anschaulich machen. Diese These möchten wir anhand konkreter Passagen des Tutorial-Videos erläutern, wobei wir uns auf die visuelle Ebene des Zeigens konzentrieren.
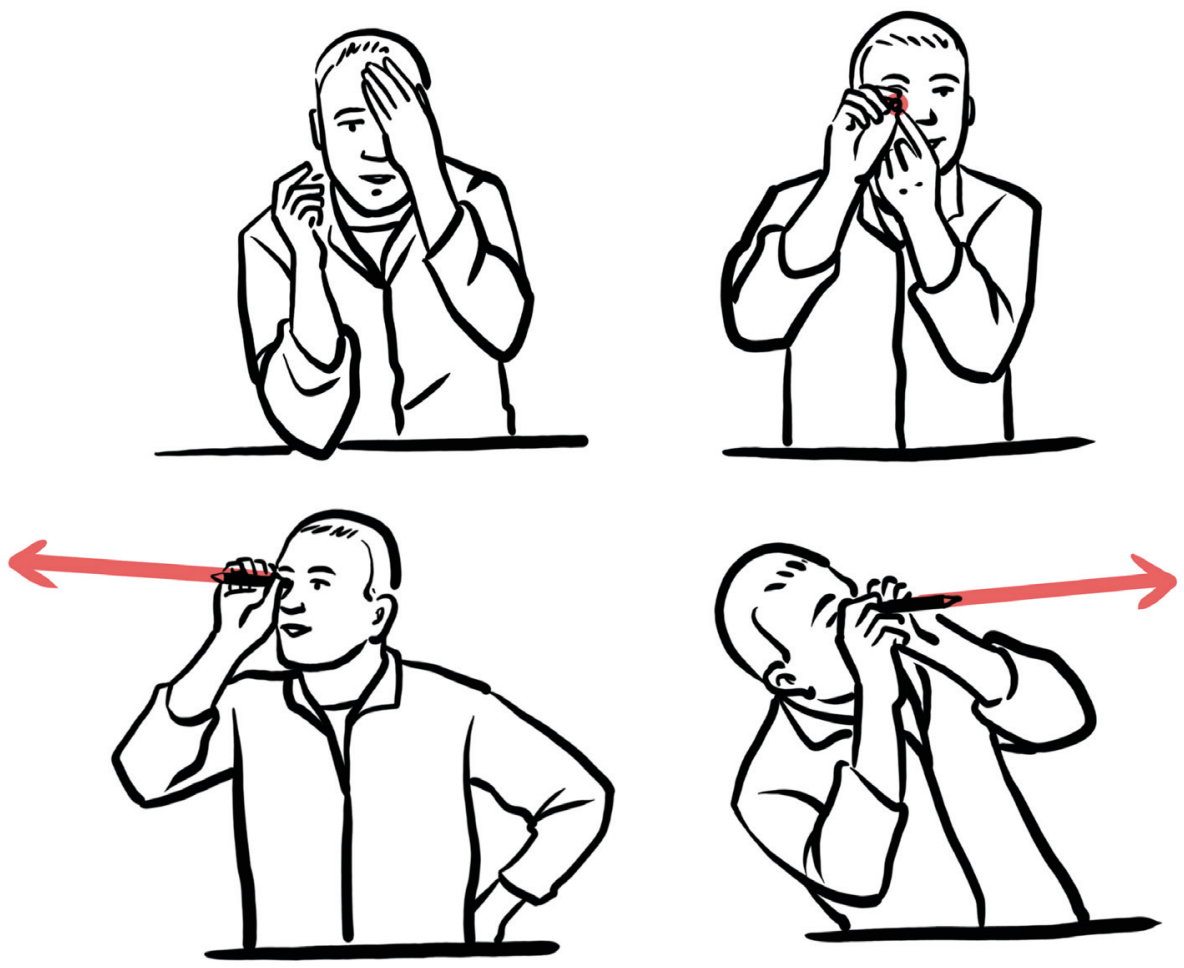

Abb. 19: Performativ verstärkte Demonstration der Subjektgebundenheit des Sehens (Visualisierungen zu Standbildern aus dem Tutorial-Video «Der Fluchtpunkt beim Zeichnen», Yadegar Asisi, 2020, Min. 07:18, 07:46, 07:48 und 08:08, Nadia Bader). 
Zunächst erscheint uns aufschlussreich zu betrachten, wie sich Asisi als aktiv handelnder Betrachter und 〈Perspektivenerzeuger〉 mit-thematisiert. Asisi sitzt in dieser Szene an einem Tisch im Atelier (ab Min. 07:18, Abb. 19). Indem er mit der Hand sein linkes Auge verdeckt, nimmt er eine erste Veranschaulichung und Vereinfachung vor, um perspektivisches Sehen zu erklären: von einer beidäugigen, stereoskopischen Sicht hin zum einäugigen Sehen. In einem nächsten Schritt nimmt Asisi einen Bleistift zur Hand, den er vor sein rechtes Auge hält: eine Veranschaulichung des «Sehstrahls», der von seinem Auge ausgeht (vgl. Platon). Daran anschliessend performt Asisi den Sehvorgang, indem er seinen Blick - mit dem Bleistift als Richtungsweiser - im Raum herumschweifen lässt, wobei er nicht nur seinen Kopf sondern seinen ganzen Oberkörper deutlich bewegt. Als Zuschauende sehen wir, wohin Asisi blickt, ohne zu sehen, was er sieht. Die Standortgebundenheit des (eigenen) Sehens wird so als solche anschaulich gemacht auf körperlicher, leiblicher und auf zeitlicher Ebene sowie durch einen Entzug, ein Nicht-Sehen-Können. Es wird also keine Perspektivübernahme im Sinne einer impliziten Setzung und stillschweigenden medialen Vorannahme suggeriert (vgl. Tutorial 2), sondern vielmehr wird der Vorgang, das Herstellen eines gerichteten Blickens explizit anschaulich gemacht.

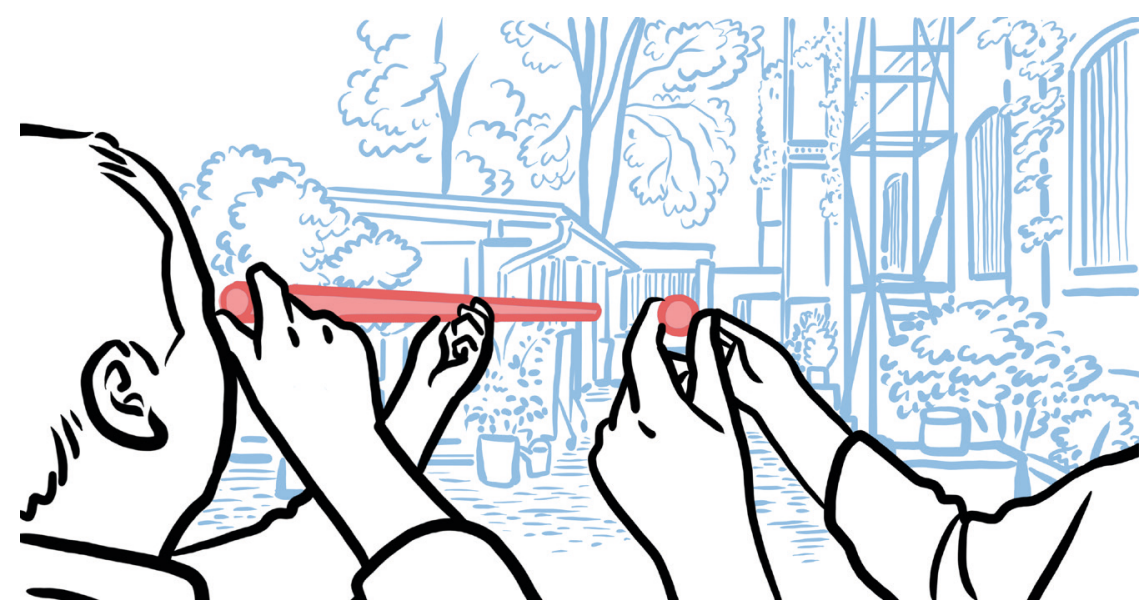

Abb. 20: Versuch einer Perspektivübernahme (Visualisierungen zu Standbildern aus dem Tutorial-Video «Der Fluchtpunkt beim Zeichnen», Yadegar Asisi, 2020, Min. 16:48, 17:47 und 18:47, Nadia Bader). 
In der übernächsten Szene (ab Min. 15:26) lädt uns Asisi zu einer Perspektivübernahme ein. Er nimmt sein Kamerateam mit nach draussen in einen Hinterhof und greift zu einem Besenstiel, der ihm zur Veranschaulichung (s)eines Sehstrahls dient - im Vergleich zum Bleistift nun in vergrösserter Skala. Im Zuge seiner Ausführungen adressiert er uns als Rezipierende in zweifacher Weise: einerseits als Zuschauende dessen, wie er vor der Kamera entlang des Besenstiel-Sehstrahls in den Hinterhof blickt und andererseits als Schauende, die nun selbst durch das Auge der Kamera in die Tiefe des videografierten Raums blicken (vgl. Abb. 20).

So wird (unsere) Wahrnehmung als subjekt- und standortgebundene Wahrnehmung explizit thematisiert, was wir als medienkritische ästhetische Praxis ansehen. Damit geht keine interessensgeleitete, thematische Subjektorientierung, sondern eine medial voraussetzungsvolle Orientierung am Subjekt als Ausgerichtetem einher. Ausrichten als Prozess zu verstehen bedeutet auch, dass es - einer praxistheoretischen Logik folgend - Praktiken des Suchens, Schärfens, Verschiebens, Vergessens usw. gibt, also kein zeitlich unverändert überdauerndes, starres Bezugssystem.

Neben der Einladung zum spielerischen Umgang mit perspektivischen Ausrichtungen verweist das Tutorial-Video von Asisi auch auf ein besonderes Potenzial des Imperfekten. Die eher zufällig, vielleicht etwas «unprofessionell» wirkende Situation im Hinterhof erscheint uns interessant, da damit nicht von einer extra vorbereiteten, modellhaft besonders zugerichteten Umgebung, sondern vielmehr von einer Alltagssituation ausgegangen wird, wie sie vielerorts anzutreffen ist. Asisi nutzt diese Situation, um seinem Kamerateam und uns als Rezipierenden etwas zu zeigen - inklusive aller Ungenauigkeiten beim etwas wackeligen, demonstrativen Hinhalten des Besenstils vor die Kameralinse - und so aus einem anschaulichen Handeln heraus der Frage nach einem perspektivischen Sehen weiter nachzugehen. Dieses induktive Vorgehen kann als ein medienkritisches Vorgehen betrachtet werden. Zahn spricht u. a. von einem medialen (Inter-) Agieren, einer involvierenden Nähe und Immersion (Zahn 2020, 216), was uns im Fall von Asisis (medialer) Vermittlungspraxis durchaus gegeben zu sein scheint. Das Investieren von erfahrbarer Zeit (z. B. im Prozess der Perspektivübernahme über den Besenstiel) erscheint hier wesentlich dafür zu sein, damit sich die medienkritischen und dadurch bildenden Potenziale 
im Tutorial-Video-Format entfalten können. Statt die gemachten Erfahrungen Anderer komprimiert als Lösung oder Fehler darzustellen, macht sich hier jemand nicht nur kognitiv, sondern in medial vermittelter, doch zugleich leibsinnlich nachvollziehbarer Präsenz die Mühe, ein Verstehen zu ermöglichen, das in immer neue Kontexte einbettet wird.

Im Video offenbart sich dadurch medial sowie inhaltlich eine medienkritische Professionalität, da unterschiedliche Perspektiviertheiten mitgedacht werden. Entsprechend wechseln beispielsweise räumliche Situationen und Orte, Darstellungsmedien, Stile und Methoden. Betrachtende müssen sich selbst immer wieder neu über verschobene Fluchtpunkte, Einstellungsgrössen und Brennweiten auf das zu sehen Gegebene ausrichten. Aktiv können sie im Medium des Videos diesen Prozess durch das interessegeleitete Scrollen (Fast Forward, Pause, Wiederholung) unterstützen, welches deswegen nicht nur eine temporale, sondern auch eine identifizierende Rolle spielt. Das Tutorial-Video bietet genügend Anregungen, um einen eigenen Zugang zu entwickeln.

Resümierend lässt sich ein Investieren in die Wahrnehmung erkennen, ein sukzessives Sich-Annähern an Seherfahrungen im perspektivischen Dispositiv, das nicht kognitiv abgekürzt werden kann: Im Sinne einer transformatorischen Bildungstheorie sind Wahrnehmungsbrüche, die zum Beispiel als formale Stilbrüche bei Asisi schnell auszumachen sind, potenziell ermächtigender als etwa die gelernte und unhinterfragt reproduzierte symbolische Form der Perspektive selbst.

\section{Chance des Imperfekten: Sensibilisierung für mediale Oberflächen und ihre Brüchigkeit}

Die medienästhetische Frage nach dem Wie und Wodurch der Darstellung, die unsere Ausführungen wie ein roter Faden durchzieht, sollte eine medial sensibilisierte, repräsentationskritische Sichtweise eröffnen. Dabei erweist sich gerade die Methode, keine perfekten, hermetisch geschlossenen Oberflächen zu produzieren, als anregend für Rezipierende. Kunstwerke, wie «Sunscreenpill» von Sandra Vaka Olsen (2014) (Abb. 2), können auf rezeptionsästhetischer Ebene verdeutlichen, wie die (schauenden) Subjekte sich mit all ihrem Begehren und ihren blinden Flecken im Bild wiederfinden. 
Beispielsweise schiebt sich bei Olsen die inszenierte indexikalische Spur eines schmutzigen Fingers immer wieder störend ins Bewusstsein. So entpuppt sich die 〈fehlerhafte〉 Nachlässigkeit gerade in der Bildstörung als bildend, weil sie den Fokus der Wahrnehmung zwischen Material- und Bildförmigkeit umspringen lässt (vgl. Zahn 2013). Durch die schmutzige Oberfläche offenbart sich ebenfalls die raum-zeitliche Dimension, die das Bild als (materielles) Ding mit imaginärem Überschuss thematisiert.

Wenn Bildung an einen konkret erfahrbaren Zeit-Raum gekoppelt ist, der sich auch während des Scrollens innerhalb unserer PDF-Datei zeigt, möchten wir uns abschliessend die Frage stellen, wie sich digitale Zeiträume in materiell involvierende Zeit-Räume womöglich zurückverwandeln lassen. Als letztes Experiment soll die kommende Seite in Schwarz (als Hintergrund und Projektionsfläche) den Lesevorgang als Wahrnehmungsvorgang 〈spiegeln〉 und dessen hermetisch mediale Oberfläche durchbrechen und invertieren. Ins Bildhafte überführt dient die folgende ins Schwarz invertierte Seite der Sichtbarmachung ganz anderer Sichtbarkeiten, die wir als Autorinnen antizipieren. Denn machen sich nun nicht die Flusen und Flecken auf dem eigenen Bildschirm bemerkbar? (vgl. Schmidt-Wetzel 2007, «0. T. (schwarz/weiß)»)

So schafft es abschliessend eine Bildform über den eintretenden spiegelnden Effekt, Sie als Subjekte in ihrer Wahrnehmungsdisposition anders auszurichten und Ihre medial bedingte Standortgebundenheit vor dem Bildschirm in unser Bild-Text-Gefüge zu integrieren. In diesem Sinne liegt folgende letzte Grenzüberschreitung nahe, zum Beispiel die Materialität des digitalen Displays noch weiter zu erkunden, und mit dem fettigen Finger mal eben... 


\section{Literatur}

AkademieRuhr. 2017. «TOP 5 Fehler beim Zeichnen. Ein Muss für jeden, der richtig zeichnen lernen möchte!» [Video 1 von 5, Fehler Nr. 1: Perspektive]. https:// www.youtube.com/watch?v=rZOczWwByvM [Zugriff: 24.08.2021].

Asisi, Yadegar. 2020. «Der Fluchtpunkt beim Zeichnen: Einfach erklärt | Sehen \& Gestalten \#ll mit Yadegar Asisi». https://www.youtube.com/ watch?v=WCbfdRQIV5U [Zugriff: 24.08.2021].

Bader, Nadia. 2022 im Erscheinen. «Zeichnenlernen online. Kunstdidaktische Betrachtung eines Tutorial-Videos zum perspektivischen Zeichnen». Herausgegeben von Thomas Gisler, Nadia Bader, Cindy Heller, Doris Signer, Mélanie Tanner, und Verena Widmaier. Heft 15 (Ohne Worte).

Bader, Nadia. 2020. "Angesammelt, Eingeordnet, Ab- oder Hingestellt | Fachschaftsräume im Bildnerischen Gestalten nachzeichnen». Herausgegeben von Verena Widmaier, Mélanie Tanner, Thomas Gisler, Cindy Heller, und Anina Meier. Heft 13 (Nachlass): 16-31.

Bader, Nadia. 2019. Zeichnen - Reden - Zeigen. Wechselwirkungen zwischen LehrLern-Dialogen und Gestaltungsprozessen im Kunstunterricht. München: kopaed.

Bader, Nadia, und Michaela Götsch. 2020. «Mit der Kamera im Rücken und dem Stift auf der Wand. Wie können ästhetische Erfahrungen Handlungskompetenzen an digital-analogen Schnittstellen fördern?». BÖKWE I (März): 27-37. http://www.boekwe.at/wp-content/uploads/2020-ol.pdf.

Blum, Gerd, und Johan Frederik Hartle. 2010. «Zelle, Raster, Würfel. Überlegungen zu Michel Foucault, Peter Halley und Gregor Schneider». In Cella. Strukturen der Ausgrenzung und Disziplinierung, herausgegeben von Christoph Bertsch und Silvia Höller, 207-215. Innsbruck: Studienverlag.

Böhme, Katja. 2018. «Was der Raum über den anderen erzählt... Fotografische Darstellungen vom 〈Räumen〉 als Grundlage einer Reflexion (kunst)pädagogischer Fragen». In räumen - Raumwissen in Natur, Kunst, Architektur und Bildung, herausgegeben von Birgit Engel, Helga Peskoller, Kirstin Westphal, Katja Böhme, und Simone Kosica, 125-53. Weinheim: Beltz Juventa.

Brandstätter, Ursula. 2013. Erkenntnis durch Kunst: Theorie und Praxis der ästhetischen Transformation. Köln: Böhlau.

Brian thekone99o. 2014. «Tutorial Zeichnen lernen - 2 Fluchtpunkt Perspektive». https://www.youtube.com/watch?v=jswknMPx308 [Zugriff: 24.08.2021].

Crary, Jonathan. 1992. Techniques of the observer. Cambridge/Massachusetts: The MIT Press.

Dorgeloh, Stephan, und Karsten D. Wolf, Hrsg. 2020. Lehren und Lernen mit Tutorial und Erklärvideos. Weinheim: Beltz.

Dürer, Albrecht. 1525. «Der Zeichner der Laute». In Underweysung der Messung, mit dem Zirckel und Richtscheyt, in Linien ebnen un[d] gantzen Corporen, von Albrecht Dürer. Nürnberg, s.n., 168. München, Bayerische Staatsbibliothek. https://www.digitale-sammlungen.de/de/view/bsbo0084858?page=168,169. 
Hitchcock, Alfred. 1954. Das Fenster zum Hof [ Rear Window ]. Patron Inc. / Paramount Pictures. 4K-UHD: Universal, 2020.

Hockney, David. 1985. Paint Trolley, L.A., fotografische Collage, 41x61 in., https:// www.hockney.com/works/photos/photographic-collages.

Hoffmann, Katja. 2015. "Jenseits der Zentralperspektive. Zum Dispositiv der räumlichen Darstellung im Zeichenunterricht - und ihren Alternativen». In Staub aufwirbeln. Eine Anleitung zum Zeichnen lehren für die kunstpädagogische Praxis, herausgegeben von Stefanie Marr, 357376. Oberhausen: Athena.

Johns, Stefanie. 2021. Vom Zwischen aus. Weisen bildreflexiver Annäherungen an Bilderfahrung in Wissenschaft, Kunst und Vermittlung. München: kopaed.

Jörissen, Benjamin, und Torsten Meyer, Hrsg. 2015. Subjekt Medium Bildung. Wiesbaden: Springer VS. https://doi.org/10.1007/978-3-658-06171-5.

Karl, Notburga. 2022 im Erscheinen. «In Malewichs Weiß ist Gelb enthalten, weil sich dahinter die Erinnerung an Gold verbirgt): Überlegungen zum Zusammenhang zwischen Kunstpraxis und Kunstpädagogik». In Kunstpädagogik und... Bezugsfelder und Perspektiven kunstpädagogischer Theorie und Praxis, herausgegeben von Sara Hornäk und Susanne Henning, 54-76. Oberhausen: Athena.

Karl, Notburga. 2020. «Zeichnen als formende Sensibilisierung: Was Körper von Körpern lernen können». Zeitschrift ästhetische Bildung 12 (1). http://zaeb.net/ wordpress/wp-content/uploads/2020/05/Karl_April-20CR-kleiner.pdf.

Karl, Notburga. 2014. "I...es inspiriert mich auch irgendwie immer wieder.) Das Bildhafte als Reflexionsebene im Werkstattbuch von Pia G.». Zeitschrift für ästhetische Bildung 6 (2). http://zaeb.net/wordpress/wp-content/ uploads/2020/12/95-377-1-PB.pdf.

Karl, Notburga. 2021. Stepping into the Picture: Bild und Performance in Joan Jonas' 〈The Shape, the Scent, the Feel of Things〉 (2005-12). University of Bamberg Press. https://doi.org/10.20378/irb-49662.

Koller, Hans-Christoph. 2018. Bildung anders denken. Einführung in die Theorie transformatorischer Bildungsprozesse. Stuttgart: Kohlhammer.

Laleg, Dominique. 2021. Kritik der Perspektive. Zürich: Diaphanes.

Loemke, Tobias. 2019. Innehalten beim Begleiten künstlerischer Prozesse: Handlungsleitende Orientierungen im Ausbreiten von Artefakten und Erzählen von Ereignissen. Erlangen: FAU Press. https://doi.org/10.25593/978-3-96147-128-7.

Lüthy, Michael. 2012. «Das Medium der ästhetischen Erfahrung: Wittgensteins Aspektbegriff, exemplifiziert an Pollocks Malerei». In Imaginäre $\mathrm{Me}$ dialität - Immaterielle Medien, herausgegeben von Gertrud Koch, Kirsten Marr, und Fiona McGovern, 125-142. München: Wilhelm Fink. https://doi. org/10.30965/9783846752371_009.

Marr, Stefanie, Hrsg. 2015. Staub aufwirbeln. Eine Anleitung zum Zeichnen lehren für die kunstpädagogische Praxis. Oberhausen: Athena. 
Mersch, Dieter. 2014. «Die Zerzeigung: Über die 〈Geste〉 des Bildes und die 〈Gabe〉 des Blicks». In Bild und Geste: Figurationen des Denkens in Philosophie und Kunst, herausgegeben von Ulrich Richtmeyer, Fabian Goppelsröder, und Toni Hildebrandt, 15-44. Bielefeld: transcript. https://doi.org/10.1515/ transcript.9783839424742.15.

Modzelewski, Andreas M. 2021. 100 geniale Zeichentricks. Von der Hilfslinie bis zur Szenerie. Igling: EMF.

Panofsky, Erwin. 1927. «Die Perspektive als 〈symbolische Form»». In Aufsätze zu Grundfragen der Kunstwissenschaft, herausgegeben von Erwin Panofsky, H. Oberer, und E. Verheyen. Berlin: Spiess.

Pazzini, Karl-Joseph. 1992. Bilder und Bildung. Vom Bild zum Abbild bis zum Wiederauftauchen der Bilder. Münster/Hamburg: Lit.

Sabisch, Andrea. 2019. «Responsivität und Medialität in Bildungs- und Erfahrungsprozessen». In Irritation als Chance. Bildung fachdidaktisch denken, herausgegeben von Ingrid Bähr, Gebhard Ulrich, Krieger Claus, Lübke Britta, Malte Pfeiffer, Tobias Regenbrecht, Andrea Sabisch, und Wolfgang Sting, 105132. Wiesbaden: Springer. https://doi.org/10.1007/978-3-658-20293-4_3.

Schmidt-Wetzel, Miriam. 2007. o. T. (schwarz/weiß). Video-Installation, 3 VideoBeamer, 3 DVD-Player, s/w, 59:52 Min. (Loop). http://schmidt-wetzel.de/msw_ medien.html.

Schnurr, Ansgar. 2015. «Forschen mit Kontrastmittel. Über die Grenzen ästhetischer Erfahrung». In Kunst Pädagogik Forschung: Aktuelle Zugänge und Perspektiven, herausgegeben von Torsten Meyer und Andrea Sabisch, 103-112. Bielefeld: transcript. https://doi.org/10.1515/9783839410585-006.

Steyerl, Hito. 2015. Factory of the Sun. Environment mit Videoinstallation, ๑ Artist and Andrew Kreps Gallery, New York. https://www.moca.org/exhibition/hitosteyerl-factory-of-the-sun.

Trecartin, Ryan, und Lizzie Fitch. 2009-10. The Re'Search (Re'Search Wait'S), HD video, 40:06 min @ Andrea Rosen Gallery, https://vimeo.com/24631059.

Vaka Olsen, Sandra. 2014. Sunscreenpill. Analoger C-Print mit SonnenschutzFotogramm auf Dibond, Acrylglasrahmen, @ Kunstverein Nürnberg. https:// kunstvereinnuernberg.de/en/editions/sunscreenpill-2014.

Zahn, Manuel. 2020. «Ästhetische Praxis als Kritik: Vom Aussetzen des Urteilens und der Empfindung neuer Wahrnehmungs-, Denk- und Handlungsmöglichkeiten». In Digitalisierung - Subjekt - Bildung, herausgegeben von Valentin Dander, Patrick Bettinger, Estella Ferraro, Christian Leineweber, und Klaus Rummler, 213-233. Obladen: Verlag Barbara Budrich. https://doi.org/10.2307/j. ctvvb7n3h.14.

Zahn, Manuel. 2013. «Das staunende Sehen im Blick des Films. Von Perzepten, Zeichen und (dem) Wundern». In Das Unverfügbare. Wunder, Wissen, Bildung, herausgegeben von Karl-Josef Pazzini, Andrea Sabisch, und Daniel Tyradellis, 199-210. Zürich: Diaphanes. 